\begin{tabular}{|c|l|}
\hline Title & Beauty Contests and A sset Prices under A symmetric Information \\
\hline Author(s) & Ishikawa, Ryuichiro; Kudoh, Noritaka \\
\hline Citation & Discussion Paper, Series A, 218, 1-30 \\
\hline Issue Date & 2010-01-24 \\
\hline Doc URL & http://hdl.handle.net/2115/42576 \\
\hline Type & bulletin (article) \\
\hline File Information & DPA 218.pdf \\
\hline
\end{tabular}

Instructions for use 
Discussion Paper, Series A, No. 2010-218

\title{
Beauty Contests and Asset Prices under Asymmetric Information
}

\author{
Ryuichiro Ishikawa and Noritaka Kudoh
}

January, 2010

Graduate School of Economics \& Business Administration Hokkaido University

Kita 9 Nishi 7, Kita-Ku, Sapporo 060-0809, JAPAN 


\title{
Beauty Contests and Asset Prices under Asymmetric Information*
}

\author{
Ryuichiro Ishikawa $^{\dagger}$ \\ Noritaka Kudoh ${ }^{\ddagger}$ \\ University of Tsukuba \\ Hokkaido University
}

January 24, 2010

\begin{abstract}
In this paper, we study a dynamic Gaussian financial market model in which the traders form higher-order expectations about the fundamental value of a single risky asset. Rational uninformed traders are introduced into an otherwise standard differential information economy to investigate the impact of asymmetric information. In a two-period economy, there is a unique linear equilibrium; beauty contests under asymmetric information do not introduce excess volatility driven by self-fulfilling multiple equilibria. Under certain conditions, there is a nonmonotonic relationship between price volatility and the proportion of uninformed traders.
\end{abstract}

JEL classification: D82, D84, G12, G14

Keywords: higher-order expectations, asset prices, asymmetric information

${ }^{*}$ We thank Katsutoshi Wakai for his insightful comments and encouragement. We also thank Katsumasa Nishide, Akira Yamazaki, Kazuhiko Ohashi, Atsushi Kajii, Shingo Takagi, and participants of FESAMES in Tokyo and seminars at GRIPS, Yokohama National University, Niigata University, Kyoto University Institute of Economic Research, and Hokkaido University for their helpful comments.

${ }^{\dagger}$ Department of Social Systems and Management, Graduate School of Systems and Information Engineering, University of Tsukuba, 1-1-1, Ten-noudai, Tsukuba, Ibaraki 305-8573 JAPAN; Email: ishikawa@sk.tsukuba.ac.jp

${ }^{\ddagger}$ Corresponding author: Department of Economics, Hokkaido University, Kita 9 Nishi 7, Kita-ku, Sapporo 0600809, JAPAN; Email: kudoh@econ.hokudai.ac.jp 


\section{Introduction}

Current and previous financial turmoil stimulates lively debates about the efficiency of financial markets in aggregating information and the "correct" pricing of assets. Before the recent subprime crisis, the dominant view among economists was that financial markets are largely efficient, and this view is summarized as the efficient markets hypothesis. ${ }^{1}$ On the other hand, recently, researchers have explored the possibility of the persistent and systematic mispricing of assets. The efficient markets hypothesis, in part, explained why macroeconomists and central bankers could safely ignore the financial markets when designing policies. ${ }^{2}$ Thus, the issue of market efficiency is more important than ever because its relevance extends to related fields of study.

In his General Theory, Keynes (1936) pointed out that higher-order expectations (HOE) are the key to understanding the stock market, and this view is often referred to as the beauty contests view of the stock market. Despite its popularity, there has been little progress in modeling beauty contests until recently. This is because analyzing the HOE of traders is difficult.

Building on the standard noisy rational expectations model of financial markets, Allen, Morris, and Shin (2006, hereafter AMS) developed a model of the stock market with overlapping generations (OLG) in which risky assets change hands as time passes. ${ }^{3}$ The final group of traders is only concerned with the fundamental value of the risky asset. However, in the period just before the final round of trade, traders need to form expectations about the expectations held by the traders of the next generation about fundamentals. This is because traders must sell their assets to the traders of the next generation. Thus, the OLG structure naturally gives rise to a framework for studying HOE in financial markets. ${ }^{4}$ AMS established that in such an environment, the law of iterated expectations fails, thereby giving rise to systematic mispricing.

\footnotetext{
${ }^{1}$ See LeRoy (1989) for a brief overview of this extensive literature.

${ }^{2}$ See, for example, "The other-worldly philosophers" and "Efficiency and beyond" The Economist, July 18 th 2009.

${ }^{3}$ Early contributions to noisy rational expectations models include those of Grossman (1976), Grossman and Stiglitz (1980), Hellwig (1980), and Diamond and Verrecchia (1981). Extremely useful surveys are available in Brunnermeier (2001), Chamley (2004), and Vives (2008).

${ }^{4}$ The OLG structure is often employed in the theory of fiat money precisely because of the property that money changes hands. See Samuelson (1958) on this point.
} 
Keynes (1936, p. 154) observed, "A conventional valuation which is established as the outcome of the mass psychology of a large number of ignorant individuals is liable to change violently as the result of a sudden fluctuation of opinion due to factors which do not really make much difference to the prospective yield; since there will be no strong roots of conviction to hold it steady." In modern terminology, he argued that a large number of uninformed traders can induce self-fulfilling multiple equilibria. Thus, it is reasonable to ask whether beauty contests are destabilizing when there is a large number of "ignorant" individuals. In the AMS model, information is differential but symmetric in the sense that each rational trader receives a signal with the same precision. In this paper, we introduce rational uninformed traders into the AMS economy to investigate whether asymmetric information introduces self-fulfilling multiple equilibria.

The structure of our model is similar to the AMS model. There is a single risky asset, whose fundamental value (or, the liquidation value) is revealed only after the final trading round, after which all traders liquidate the asset. The presence of the final trading round suggests that, under complete information, backward induction dictates and there cannot be any systematic mispricing, or bubble, as shown by Tirole (1982). ${ }^{5}$ The importance of the finite-horizon assumption is that it makes bubbles impossible under complete information; thus, this assumption clarifies the role of incomplete information. ${ }^{6}$ Of related interest, an advantage of a model with a finite horizon is that the fundamental value of the risky asset is easily defined, which in turn implies that it is much easier to define the bias of the asset price.

Following AMS, we consider a dynamic stock market economy in which there are overlapping generations of two-period-lived traders so that beauty contests arise naturally. ${ }^{7}$ In each generation, there are informed traders, uninformed traders, and noise traders. Noise traders prevent prices from fully revealing (Grossman and Stiglitz, 1980). Thus, we seek a noisy rational expectations

\footnotetext{
${ }^{5}$ For this reason, the macroeconomics literature employs infinite-horizon models to study bubbles (Tirole, 1985).

${ }^{6}$ Early contributions to modeling bubbles in a finite-horizon economy include Allen and Gorton (1993) and Allen et al. (1993).

${ }^{7}$ According to Keynes (1936, pp. 154-155), professional investors and speculators "are concerned, not with what an investment is really worth to a man who buys it "for keeps", but with what the market will value it at, under the influence of mass psychology, three months or a year hence."
} 
equilibrium (NREE) under limit orders, in which all rational traders submit their asset demand functions to the market. To rule out strategic interactions among traders (Kyle, 1989), we assume that traders are atomless.

Recently, a literature exploring the issue of multiple equilibria in noisy rational expectations model of the stock market has developed. Cespa (2002), for example, found that short horizon combined with serially correlated noise trading leads to multiple linear equilibria. Cespa and Vives (2007) generalized the model of Cespa (2002) to clarify the relationship between the serial correlation of noise trading and the likelihood of equilibrium multiplicity. Our framework is a special case of those of Cespa (2002) and Cespa and Vives (2007) in the sense that there is no serial correlation in noise trading, as assumed by AMS.

Under symmetric (but differential) information, the absence of serial correlation leads to a unique equilibrium, as shown in other studies. However, under asymmetric information, the uniqueness of equilibrium is not trivial. Notable examples are Gennotte and Leland (1990), and more recently, Barlevy and Veronesi (2003), who showed in their static models that there are multiple NREE under asymmetric information. This occurs because uninformed traders (i.e., market makers) are not certain whether they are trading against noise traders or informed traders.

We show that there is a unique linear NREE in our two-period economy. According to our results, beauty contests under asymmetric information do not introduce self-fulfilling multiple equilibria. This suggests that for equilibrium multiplicity, one needs to introduce either serial correlation in noise trading or nonlinear behavior among noise traders. Given our uniqueness result, we obtain an exact solution. Since the linear price equations have coefficients that are extremely complicated, we present a number of numerical results.

We replicate one of the main results of AMS that the information content of the asset price increases over time (see also He and Wang, 1995). We also verify that the information content of the price increases with the proportion of informed traders. Interestingly, the price's reliance on the current public belief decreases with the proportion of informed traders. The price's reliance on the public belief decreases over time unless the information content of the price in the first period 
is sufficiently high. This occurs when the private signals are highly informative and the proportion of informed traders is sufficiently high.

In the spirit of Wang (1993), we study the impact of asymmetric information on asset price volatility. We proceed by studying two measures, market liquidity and the variance of the difference between two consecutive prices. Market liquidity captures the response of the asset price to a supply shock driven by noise trading. Price volatility depends on market liquidity and on the variation in private information.

In the benchmark economy, in which the private signals have the same precision as do prior public information and noise trading, market liquidity increases both with the proportion of informed traders, and over time. In this case, price volatility decreases with the proportion of informed traders. In other words, "ignorant" traders are destabilizing.

However, we find examples in which "ignorant" traders are stabilizing. This occurs when market liquidity decreases over time. An increase in the variance of the price in the next period lowers market liquidity. We find that market liquidity decreases over time if either the private signals are noisy or there is a small proportion of informed traders with precise information. We also find an example in which there is a nonmonotonic relationship between price volatility and the proportion of uninformed traders.

The results described above are derived under the assumption of limit orders, under which all rational traders submit their demand functions to the market. How robust are the results to changes in market microstructure? ${ }^{8}$ To address this issue we develop a model with market orders in which the informed traders submit quantities of assets demanded, rather than functions, to the market. By using the same set of parameters as the model with limit orders, we establish that there is a unique NREE for each level of the proportion of informed traders. As expected, the information content of the price is generally lower under market orders than under limit orders. Interestingly, for many parameter values, asset price volatility is lower under market orders than under limit orders.

The rest of the paper is organized as follows. In Section 2, we develop the model and derive

\footnotetext{
${ }^{8}$ For a review of market microstructure, see O'Hara (1995), Brunnermeier (2001), and Vives (2008), among others.
} 
the asset pricing formula. In Section 3, to provide a benchmark, we solve the static version of the model. Since the analysis of the general multiperiod economy is quite complicated, in Section 4, we focus on a model with two trading periods. In Section 5, we obtain results under market orders. Section 6 concludes the paper. The Appendix includes proofs of propositions. 


\section{The Model}

\subsection{Environment}

Time is discrete. There is a single risky asset with the liquidation value $\theta$, which is normally distributed with mean $y$ and variance $1 / \alpha$. There is also a risk-free asset with zero net return. Following AMS, we assume that the risky asset is traded at dates $t=1, \ldots, T$. The realization of the liquidation value $\theta$ is determined at $t=0$, but can be liquidated only at date $T+1$.

Traders are a sequence of two-period-lived overlapping generations. At each date, a new generation with a unit measure is born. Two immediate implications are worth emphasizing: one is that the order of play is sequential and fixed by date of birth, which rules out the issues of delay (Chamley and Gale, 1994) and herding as a result of the clustering of decisions (Chari and Kehoe, 2004); the other is that traders, except for those from the final generation, must sell their assets to other traders, which forces them to form HOE.

Following AMS, we introduce noise traders to prevent the current price to be fully revealing (Grossman and Stiglitz, 1980). In any period, there are four types of traders: young traders, old traders, young noise traders, and old noise traders. Since traders live for only two trading rounds, all traders unwind their entire positions when old with certainty. In other words, the only source of uncertainty is the young noise traders. The behavior of the young noise traders, who are not utility maximizers, is summarized by a noisy net supply of the risky asset, $s_{t}$, which is normally distributed with mean 0 and precision $\gamma$.

Each generation consists of a unit mass of traders, each of whom is indexed by $i$. The distribution of traders is uniform on the support $[0,1]$. The key innovation of this paper is to introduce rational uninformed traders into the AMS framework. The measure of informed traders in each period is $\phi(0 \leq \phi \leq 1)$ and the measure of uninformed traders is $1-\phi$. Trader $i \in[0, \phi]$ of generation $t$ knows the full history of past and current prices as well as the realization of the private signal,

$$
x_{i t}=\theta+\varepsilon_{i t},
$$

where $\varepsilon_{i t}$ is normally distributed with mean 0 and variance $1 / \beta$. For convenience, we define trader 
$i$ 's information set in period $t$ as $\Omega_{i t}=\left\{y, p_{1}, p_{2}, \cdots, p_{t}, x_{i t}\right\}$. We assume that all uncertainty is resolved in $T+1$, so we must have $p_{T+1}=\theta$.

To simplify matters, we follow the literature in assuming that traders consume only when old. In principle, the model belongs to a class of multiperiod noisy rational expectations model (Brown and Jennings, 1990; Grundy and McNichols, 1990; He and Wang, 1995; Vives, 1995; Cespa, 2002; AMS, 2006; Cespa and Vives, 2007; Bacchetta and Wincoop, 2008). To be specific, each trader's problem is to maximize $E\left[U\left(w_{i}\right) \mid \Omega_{i t}\right]$ subject to $p_{t} X_{i t}+M_{i t}=w_{i 0}$ and $w_{i}=M_{i t}+p_{t+1} X_{i t}$, where $w_{i}$ is the level of consumption, which amounts to wealth at date $t+1, p_{t}$ is the price of the asset, $X_{i t}$ is the asset demand of trader $i$ at date $t, M_{i t}$ is the amount of money, which yields zero net return, and $w_{i 0}$ is trader $i$ 's initial wealth. Since the asset demand is known to be independent of initial wealth in a CARA-Gaussian setup, we simplify the exposition by setting $w_{i 0}=0$. The timing of events is summarized in Figure 1.

We can rewrite the trader's problem as

$$
\max _{X_{i t}} E\left[U\left(\left(p_{t+1}-p_{t}\right) X_{i t}\right) \mid \Omega_{i t}\right]
$$

We follow Grossman and Stiglitz (1980) and AMS by assuming that $U\left(w_{i}\right)=-\exp \left\{-w_{i} / \tau\right\}$, where $\tau$ is the risk-tolerance parameter. This amounts to maximizing the following:

$$
E\left[w_{i} \mid \Omega_{i t}\right]-\frac{1}{2 \tau} \operatorname{Var}\left[w_{i} \mid \Omega_{i t}\right]
$$

where $E\left[w_{i} \mid \Omega_{i t}\right]=\left[E\left[p_{t+1} \mid \Omega_{i t}\right]-p_{t}\right] X_{i t}$ and $\operatorname{Var}\left[w_{i} \mid \Omega_{i t}\right]=X_{i t}^{2} \operatorname{Var}\left[p_{t+1} \mid \Omega_{i t}\right]$. It is then easy to obtain the following asset demand function:

$$
X_{i t}=\frac{E\left[p_{t+1} \mid \Omega_{i t}\right]-p_{t}}{\tau^{-1} \operatorname{Var}\left[p_{t+1} \mid \Omega_{i t}\right]}
$$

According to (1), the trader's asset demand is increasing in the expected selling price, decreasing in risk aversion, and decreasing in the variance of the selling price.

The stock market equilibrium condition in period $t$ should be written as $\int_{0}^{1} X_{i t} d i=\int_{0}^{1} X_{i t-1} d i+$ $s_{t}-s_{t-1}$. This reflects the fact that all traders unwind their position when old. We follow AMS in assuming that the noise traders live for two periods, and unwind their positions when old. This 
implies that in the first period $(t=1)$, we have $\int_{0}^{1} X_{i 1} d i=s_{1}$. It follows that the stock market equilibrium in any period $t$ can be written as $\int_{0}^{1} X_{i t} d i=s_{t}$. Substituting (1) into this equation yields

$$
\int_{0}^{\phi} \frac{E\left[p_{t+1} \mid \Omega_{i t}\right]-p_{t}}{\tau^{-1} \operatorname{Var}\left[p_{t+1} \mid \Omega_{i t}\right]} d i+(1-\phi) \frac{E\left[p_{t+1} \mid \Omega_{t}\right]-p_{t}}{\tau^{-1} \operatorname{Var}\left[p_{t+1} \mid \Omega_{t}\right]}=s_{t}
$$

where $p_{T+1}=\theta$. This represents stock market equilibrium under asymmetric information.

Cespa (2002) and Cespa and Vives (2007) consider the case in which noise traders are not OLG traders so that the equilibrium condition is $\int_{0}^{1} X_{i t} d i=\int_{0}^{1} X_{i t-1} d i+s_{t}$ or, equivalently,

$\int_{0}^{1} X_{i t} d i=s_{t}+s_{t-1}$. Cespa and Vives (2007) investigate how this translate into multiplicity of equilibria.

\section{$2.2 \quad$ Asset Pricing Formula}

Consider the model with $T=2$. In period $T=2$, stock market equilibrium implies

$$
\phi \frac{\bar{E}\left[\theta \mid \Omega_{i 2}\right]-p_{2}}{\tau^{-1} \operatorname{Var}\left[\theta \mid \Omega_{i 2}\right]}+(1-\phi) \frac{E\left[\theta \mid \Omega_{2}\right]-p_{2}}{\tau^{-1} \operatorname{Var}\left[\theta \mid \Omega_{2}\right]}=s_{2},
$$

where $\bar{E}\left[\theta \mid \Omega_{i 2}\right] \equiv \phi^{-1} \int_{0}^{\phi} E\left[\theta \mid \Omega_{i 2}\right] d i$ is the informed traders' average expectations. Note that $\operatorname{Var}\left[\theta \mid \Omega_{i 2}\right]$ depends on $\beta$ but is independent of $i$. By solving the equilibrium condition for $p_{T}$, we obtain

$$
p_{2}=\frac{\phi \tau \kappa_{2}}{\operatorname{Var}\left[\theta \mid \Omega_{i 2}\right]} \bar{E}\left[\theta \mid \Omega_{i 2}\right]+\frac{(1-\phi) \tau \kappa_{2}}{\operatorname{Var}\left[\theta \mid \Omega_{2}\right]} E\left[\theta \mid \Omega_{2}\right]-\kappa_{2} s_{2},
$$

where $\kappa_{2}^{-1}=\phi \tau / \operatorname{Var}\left[\theta \mid \Omega_{i 2}\right]+(1-\phi) \tau / \operatorname{Var}\left[\theta \mid \Omega_{2}\right]$ is referred to as market liquidity (Kyle, 1985; He and Wang, 1995) or market depth (Vives, 2008), which equals the average of all the coefficients in the demand functions. It is easy to show that the sum of the coefficients on $\bar{E}\left[\theta \mid \Omega_{i 2}\right]$ and $E\left[\theta \mid \Omega_{2}\right]$ is unity. It is interesting to observe that even with $\phi=1 / 2$, the weight on average expectations is greater because the private belief is more precise than the public belief (i.e., $\left.\operatorname{Var}\left[\theta \mid \Omega_{i 2}\right]<\operatorname{Var}\left[\theta \mid \Omega_{2}\right]\right)$. In other words, the price equation tends to put more weight on the belief that is more precise. Under symmetric information $(\phi=1),(3)$ reduces to the AMS economy, in which $p_{2}=\bar{E}\left[\theta \mid \Omega_{i 2}\right]-$ $\tau^{-1} \operatorname{Var}\left[\theta \mid \Omega_{i 2}\right] s_{2}$. 
Consider the equilibrium in period 1 . In this trading round, traders care about the asset price in the next period $p_{2}$ rather than the fundamental value $\theta$. Stock market equilibrium in this period is given by

$$
\phi \frac{\bar{E}\left[p_{2} \mid \Omega_{i 1}\right]-p_{1}}{\tau^{-1} \operatorname{Var}\left[p_{2} \mid \Omega_{i 1}\right]}+(1-\phi) \frac{E\left[p_{2} \mid \Omega_{1}\right]-p_{1}}{\tau^{-1} \operatorname{Var}\left[p_{2} \mid \Omega_{1}\right]}=s_{1} .
$$

Solving this for $p_{1}$ yields

$$
p_{1}=\frac{\phi \tau \kappa_{1}}{\operatorname{Var}\left[p_{2} \mid \Omega_{i 1}\right]} \bar{E}\left[p_{2} \mid \Omega_{i 1}\right]+\frac{(1-\phi) \tau \kappa_{1}}{\operatorname{Var}\left[p_{2} \mid \Omega_{1}\right]} E\left[p_{2} \mid \Omega_{1}\right]-\kappa_{1} s_{1},
$$

where $\kappa_{1}^{-1}=\phi \tau / \operatorname{Var}\left[p_{2} \mid \Omega_{i 1}\right]+(1-\phi) \tau / \operatorname{Var}\left[p_{2} \mid \Omega_{1}\right]$ is market liquidity in period 1 . Note that the price equation for period 1 puts more weight on average expectations about the price in the next period $\bar{E}\left[p_{2} \mid \Omega_{i 1}\right]$ than on public expectations $E\left[p_{2} \mid \Omega_{1}\right]$. This is because private beliefs are more precise than is the public belief (i.e., $\operatorname{Var}\left[p_{2} \mid \Omega_{i 1}\right]<\operatorname{Var}\left[p_{2} \mid \Omega_{1}\right]$ ).

We substitute (3) into (4) to obtain

$$
\begin{aligned}
p_{1}= & \frac{\phi \tau \kappa_{1}}{\operatorname{Var}\left[p_{2} \mid \Omega_{i 1}\right]} \frac{\phi \tau \kappa_{2}}{\operatorname{Var}\left[\theta \mid \Omega_{i 2}\right]} \bar{E}\left[\bar{E}\left[\theta \mid \Omega_{i 2}\right] \mid \Omega_{i 1}\right]+\frac{\phi \tau \kappa_{1}}{\operatorname{Var}\left[p_{2} \mid \Omega_{i 1}\right]} \frac{(1-\phi) \tau \kappa_{2}}{\operatorname{Var}\left[\theta \mid \Omega_{2}\right]} \bar{E}\left[E\left[\theta \mid \Omega_{2}\right] \mid \Omega_{i 1}\right] \\
& +\frac{(1-\phi) \tau \kappa_{1}}{\operatorname{Var}\left[p_{2} \mid \Omega_{1}\right]} \frac{\phi \tau \kappa_{2}}{\operatorname{Var}\left[\theta \mid \Omega_{i 2}\right]} E\left[\bar{E}\left[\theta \mid \Omega_{i 2}\right] \mid \Omega_{1}\right]+\frac{(1-\phi) \tau \kappa_{1}}{\operatorname{Var}\left[p_{2} \mid \Omega_{1}\right]} \frac{(1-\phi) \tau \kappa_{2}}{\operatorname{Var}\left[\theta \mid \Omega_{2}\right]} E\left[E\left[\theta \mid \Omega_{2}\right] \mid \Omega_{1}\right]-\kappa_{1} s_{1} .
\end{aligned}
$$

Note that the period-2 price is influenced by: (i) average expectations about average expectations (about the fundamentals); (ii) average expectations about the public belief; (iii) the public belief about average expectations; and (iv) the public belief about the public belief. Thus, the higherorder terms in our formulation are more complex than those in previous studies. In particular, the case of $\phi=1$ corresponds to the AMS economy, which does not include the terms (ii)-(iv).

In each period, the price equation puts more weight on average expectations because the private belief is more precise than the public belief. This implies that as one moves away from the liquidation date, more weight is put on higher-order average expectations (such as the first term of $(4))$.

The asset pricing formula for the general $T$-period economy is much more complicated. We start with the equilibrium condition (2). To economize on notation, below we denote $\operatorname{Var}\left[\cdot \mid \Omega_{i t}\right]$ and $\operatorname{Var}\left[\cdot \mid \Omega_{t}\right]$ by $\operatorname{Var}_{i t}(\cdot)$ and $\operatorname{Var}_{t}(\cdot)$, respectively. Then, we solve $(2)$ for $p_{t}$ as

$$
p_{t}=\frac{1}{\nu_{t}}\left\{\operatorname{Var}_{t}\left(p_{t+1}\right) \bar{E}_{t}\left(p_{t+1}\right)+\frac{1-\phi}{\phi} \operatorname{Var}_{i t}\left(p_{t+1}\right) E_{t}\left(p_{t+1}\right)-\frac{\operatorname{Var}_{t}\left(p_{t+1}\right) \operatorname{Var}_{i t}\left(p_{t+1}\right)}{\phi \tau} s_{t}\right\},
$$


where $\nu_{t} \equiv \operatorname{Var}_{t}\left(p_{t+1}\right)+((1-\phi) / \phi) \operatorname{Var}_{i t}\left(p_{t+1}\right), \bar{E}_{t}(\cdot) \equiv \phi^{-1} \int_{0}^{\phi} E\left[\cdot \mid \Omega_{i t}\right] d i$, and $E_{t}(\cdot) \equiv E\left[\cdot \mid \Omega_{t}\right]$.

To proceed, we define the set of sequences of information sets as follows: $\mathcal{S}(t, k) \equiv\left\{\left\{\Omega_{* n}\right\}_{n=t}^{T}\right.$ | $\left\{\Omega_{* n}\right\}_{n=t}^{T}$ contains $k$ public information sets $\}$, where $\Omega_{* t}$ is either a private information set $\Omega_{i t}$ or a public information set $\Omega_{t}$. That is, $\mathcal{S}(t, k)$ is a collection of all sequences of information sets starting from $t$ to $T$, identified by $k$, the number of public information sets. For a given sequence $\tilde{s} \equiv\left\{\Omega_{* n}\right\}_{n=t}^{T} \in \mathcal{S}(t, k)$, we define $\mathbf{E}_{\tilde{s}}(\theta) \equiv E_{* t} E_{* t+1} \cdots E_{* T}(\theta)$ and $\operatorname{Var}_{\tilde{s}} \equiv$ $\operatorname{Var}\left(p_{t+1} \mid \Omega_{* t}\right) \operatorname{Var}\left(p_{t+2} \mid \Omega_{* t+1}\right) \cdots \operatorname{Var}\left(\theta \mid \Omega_{* T}\right)$, where $E_{* t}$ is either the expectations operator conditional on $\Omega_{t}$ from the sequence or the average expectations operator conditional on $\Omega_{i t}$ from the sequence.

Proposition 1 The asset price in period $t \leq T$ is

$$
p_{t}=\sum_{k=0}^{T-t+1} \sum_{\tilde{s} \in \mathcal{S}(T-t+1, k)} \frac{\left(\frac{1-\phi}{\phi}\right)^{k} \operatorname{Var}_{\tilde{s}} \mathbf{E}_{\tilde{s}}(\theta)}{\nu_{t} \nu_{t+1} \cdots \nu_{T}}-\kappa_{t} s_{t},
$$

where $\kappa_{t}^{-1} \equiv \phi \tau / \operatorname{Var}_{i t}\left(p_{t+1}\right)+(1-\phi) \tau / \operatorname{Var}_{t}\left(p_{t+1}\right)$ is market liquidity in period $t$.

Proof. See Appendix A.

The above asset pricing formula extends that of AMS to the case under asymmetric information. As in AMS, the price equation incorporates higher-order expectations about the liquidation value $\theta$. What differentiates our formulation from AMS's is the presence of the public expectations operator $E_{t}(\cdot)$. Under symmetric information, the price equation is relatively simple because it involves only the average expectations operator $\bar{E}_{t}(\cdot)$. Under asymmetric information, the price equation contains the expectations operators of both types. Unfortunately, the complexity of the asset pricing formula (6) prevents us from analyzing the properties of the asset price. In what follows, we proceed by limiting our analysis to the cases with $T=1$ and $T=2$.

\section{Static Equilibrium}

As a benchmark, we consider the static equilibrium in which there is only one trading round (i.e., $T=1$ ). For brevity, in what follows, we drop all time subscripts. An informed trader of type 
$i \in[0, \phi]$ purchases the asset and receives the fundamental value of the asset (such as a dividend) in the next period. Thus, the demand for the asset of type- $i$ trader is given by $X_{i}=\tau\left[E\left[\theta \mid y, p, x_{i}\right]-\right.$ $p] / \operatorname{Var}\left[\theta \mid y, p, x_{i}\right]$. Uninformed traders, on the other hand, receive no private signal. Thus, the information set for an uninformed trader is $\Omega=\{y, p\}$, which is simply public information. Thus, an uninformed trader's asset demand is given by $X^{U}=\tau[E[\theta \mid y, p]-p] / \operatorname{Var}[\theta \mid y, p]$. Stock market equilibrium is therefore given by

$$
\int_{0}^{\phi} \frac{E\left[\theta \mid y, p, x_{i}\right]-p}{\tau^{-1} \operatorname{Var}\left[\theta \mid y, p, x_{i}\right]} d i+(1-\phi) \frac{E[\theta \mid y, p]-p}{\tau^{-1} \operatorname{Var}[\theta \mid y, p]}=s .
$$

The equilibrium asset price $p$ is the solution to (7).

Proposition 2 In the static economy, $p=K(\phi) y+(1-K(\phi)) \theta-\kappa s$, where $K(\phi) \equiv \alpha /[\phi \beta+$ $\left.\alpha+(\phi \tau \beta)^{2} \gamma\right]$ and $\kappa=\left(1+\tau^{2} \phi \beta \gamma\right)\left[\phi \tau \beta+\tau \alpha+\tau(\phi \tau \beta)^{2} \gamma\right]^{-1}$; The asset demands of informed and uninformed traders are

$$
\begin{aligned}
X_{i} & =\frac{\tau \alpha}{1+\phi \tau^{2} \beta \gamma}(y-p)+\tau \beta\left(x_{i}-p\right), \\
X^{U} & =\frac{\tau \alpha}{1+\phi \tau^{2} \beta \gamma}(y-p)
\end{aligned}
$$

Proof. See Appendix B.

Since $K(\phi)$ is decreasing, the asset price reflects the fundamental value more precisely the higher is the proportion of informed traders. In other words, the information content of the price increases as the proportion of informed traders increases.

The demand function (8) is interpreted as follows. The first term represents the trader's market making position. The coefficient is increasing in the risk-adjusted public information quality, $\tau \alpha$, and is increasing in the volume of noise trading. ${ }^{9}$ The second term represents the speculative position of the informed trader; he or she reacts to the difference between his or her private information (or judgment) and the price. The coefficient $\tau \beta$ represents the risk-adjusted informational advantage of the informed trader (Vives, 2008).

As is evident from (9), the uninformed trader's asset demand has only one component: the market making position. The market making position is decreasing in $\phi$ because of the adverse

\footnotetext{
${ }^{9}$ The expected volume of noise trading $E[|s|]$ is proportional to its variance $\gamma^{-1}$. See Vives (2008).
} 
selection problem. If $\phi$ is small, then uninformed traders feel safe in the sense that they are less likely to be trading against the informed.

The price equation $p=K(\phi) y+(1-K(\phi)) \theta-\kappa s$ implies that the volatility of the price is given by $\operatorname{Var}[p]=(1-K(\phi))^{2} / \alpha+\kappa^{2} / \gamma$. The first term increases as the information content of the price increases. This comes from the diversity of information. The second term comes from the random supply shock. In the existing literature, $\kappa^{-1}$ is market liquidity, which increases as the slope of the aggregate asset demand curve decreases. From $\kappa=\left(1+\tau^{2} \phi \beta \gamma\right)\left[\phi \tau \beta+\tau \alpha+\tau(\phi \tau \beta)^{2} \gamma\right]^{-1}$, it is easy to verify that $\partial \kappa / \partial \phi<0 \Leftrightarrow-\left(\tau^{2} \beta \gamma\right)^{2} \phi^{2}-2\left(\tau^{2} \beta \gamma\right) \phi-1+\tau^{2} \alpha \gamma=\tau^{2} \alpha \gamma-\left(\tau^{2} \beta \gamma \phi+1\right)^{2}<0$. Thus,

Proposition 3 Market liquidity is increasing in $\phi$ if and only if

$$
\phi>\bar{\phi} \equiv \frac{\sqrt{\tau^{2} \alpha \gamma}-1}{\tau^{2} \beta \gamma}
$$

There are two forces behind the result. One is that, as $\phi$ increases, the market making demand for the asset becomes less elastic, which reduces market liquidity. The other force is that, as $\phi$ increases, the speculative demand for the asset increases because the proportion of informed traders increases. This effect increases market liquidity. For parameter values that satisfy $\tau^{2} \alpha \gamma<1, \bar{\phi}$ is negative. In this case, an increase in the proportion of informed traders unambiguously increases market liquidity because the first effect is weak. Interestingly, for $\tau^{2} \alpha \gamma>1$, the first effect is sufficiently strong, so an increase in $\phi$ decreases market liquidity for small values of $\phi$.

\section{Equilibrium with Two Trading Rounds}

\subsection{Uniqueness of the NREE}

In this section, we solve the two-period version of the model. ${ }^{10}$ The solution algorithm consists of four steps: description of learning in period 1; description of learning in period 2; solving for the period-2 price; and solving for the period-1 price. Suppose that agents form a common conjecture about the period 1 price such that $p_{1}=\kappa_{1}\left(\lambda_{1} y+\mu_{1} \theta-s_{1}\right)$, from which we have

\footnotetext{
${ }^{10}$ The case with $\phi=1$ is studied in Appendix B of AMS.
} 
$z_{1} \equiv\left(p_{1}-\kappa_{1} \lambda_{1} y\right) / \kappa_{1} \mu_{1}=\theta-\frac{1}{\mu_{1}} s_{1}$. The price signal $z_{1}$ is normal with mean $\theta$ and precision $\mu_{1}^{2} \gamma$. In period 1 , after observing $p_{1}$, public beliefs about $\theta$ are updated to the normal distribution with $E\left[\theta \mid y, p_{1}\right]=\left(\alpha+\mu_{1}^{2} \gamma\right)^{-1}\left[\left(\alpha-\mu_{1} \gamma \lambda_{1}\right) y+\left(\mu_{1} \gamma / \kappa_{1}\right) p_{1}\right] \equiv y_{2}$ and $\operatorname{Var}\left[\theta \mid y, p_{1}\right]=\left(\alpha+\mu_{1}^{2} \gamma\right)^{-1} \equiv$ $1 / \alpha_{2}$. Informed traders, in addition, observe private signals. Their posterior beliefs follow the normal distribution with $E\left[\theta \mid y, p_{1}, x_{i 1}\right]=\left(\alpha+\beta+\mu_{1}^{2} \gamma\right)^{-1}\left[\left(\alpha-\mu_{1} \gamma \lambda_{1}\right) y+\left(\mu_{1} \gamma / \kappa_{1}\right) p_{1}+\beta x_{i 1}\right]$ and $\operatorname{Var}\left[\theta \mid y, p_{1}, x_{i 1}\right]=\left(\alpha+\beta+\mu_{1}^{2} \gamma\right)^{-1}$.

Similarly, suppose that agents form a common price conjecture about the period-2 price such that $p_{2}=\kappa_{2}\left(\lambda_{2} y_{2}+\mu_{2} \theta-s_{2}\right)$, where $y_{2}$ represents public expectations about the fundamentals in this period. The public signal in this period is $z_{2} \equiv\left(p_{2}-\kappa_{2} \lambda_{2} y_{2}\right) / \kappa_{2} \mu_{2}=\theta-\frac{1}{\mu_{2}} s_{2}$. The random variable $z_{2}$ is normal with mean $\theta$ and precision $\mu_{2}^{2} \gamma$, and is informationally equivalent to the price. Thus, in period 2, after observing the price, public beliefs about $\theta$ are updated to the normal distribution with $E\left[\theta \mid y, p_{1}, p_{2}\right]=\left(\alpha_{2}+\mu_{2}^{2} \gamma\right)^{-1}\left[\left(\alpha_{2}-\mu_{2} \gamma \lambda_{2}\right) y_{2}+\left(\mu_{2} \gamma / \kappa_{2}\right) p_{2}\right]$ and $\operatorname{Var}\left[\theta \mid y, p_{1}, p_{2}\right]=\left(\alpha_{2}+\mu_{2}^{2} \gamma\right)^{-1}$. The posterior beliefs of informed traders follow the normal distribution with $E\left[\theta \mid y, p_{1}, p_{2}, x_{i 2}\right]=\left(\alpha_{2}+\beta+\mu_{2}^{2} \gamma\right)^{-1}\left[\left(\alpha_{2}-\mu_{2} \gamma \lambda_{2}\right) y_{2}+\left(\mu_{2} \gamma / \kappa_{2}\right) p_{2}+\beta x_{i 2}\right]$ and $\operatorname{Var}\left[\theta \mid y, p_{1}, p_{2}, x_{i 2}\right]=\left(\alpha_{2}+\beta+\mu_{2}^{2} \gamma\right)^{-1}$.

Since we assume OLG noise traders, the market equilibrium condition for period 2 is given by

$$
\int_{0}^{\phi} \frac{E\left[\theta \mid y, p_{1}, p_{2}, x_{i 2}\right]-p_{2}}{\tau^{-1} \operatorname{Var}\left[\theta \mid y, p_{1}, p_{2}, x_{i 2}\right]} d i+(1-\phi) \frac{E\left[\theta \mid y, p_{1}, p_{2}\right]-p_{2}}{\tau^{-1} \operatorname{Var}\left[\theta \mid y, p_{1}, p_{2}\right]}=s_{2}
$$

We solve this equation for $p_{2}$ as follows:

$$
p_{2}=\frac{\phi \tau \beta \theta+\tau\left(\alpha_{2}-\mu_{2} \gamma \lambda_{2}\right) y_{2}-s_{2}}{\phi \tau \beta+\tau \alpha_{2}+\tau \mu_{2}^{2} \gamma-\tau \frac{\mu_{2} \gamma}{\kappa_{2}}} .
$$

Rational expectations require that $\kappa_{2}^{-1}=\phi \tau \beta-\tau \mu_{2} \gamma / \kappa_{2}+\tau\left(\alpha_{2}+\mu_{2}^{2} \gamma\right), \lambda_{2}=\tau\left(\alpha_{2}-\mu_{2} \gamma \lambda_{2}\right)$, $\mu_{2}=\phi \tau \beta$, from which we obtain

$$
\begin{aligned}
\kappa_{2} & =\frac{1+\tau^{2} \phi \beta \gamma}{\phi \tau \beta+\tau\left(\alpha_{2}+(\phi \tau \beta)^{2} \gamma\right)}=\frac{1+\tau^{2} \phi \beta \gamma}{\phi \tau \beta+\tau\left(\alpha+\mu_{1}^{2} \gamma+(\phi \tau \beta)^{2} \gamma\right)} \\
\lambda_{2} & =\frac{\tau \alpha_{2}}{1+\tau^{2} \phi \beta \gamma}=\frac{\tau\left[\alpha+\mu_{1}^{2} \gamma\right]}{1+\tau^{2} \phi \beta \gamma} \\
\mu_{2} & =\phi \tau \beta .
\end{aligned}
$$

Thus, $\kappa_{2} \mu_{2}=\left[\phi \beta+(\phi \tau \beta)^{2} \gamma\right] /\left[\phi \beta+\alpha_{2}+(\phi \tau \beta)^{2} \gamma\right]$, and it is easy to verify that $\kappa_{2} \mu_{2}=1-\kappa_{2} \lambda_{2}$. 
Consider the stock market equilibrium in period 1 . With the price conjecture, the public belief about $p_{2}$ is normally distributed with $E\left[p_{2} \mid y, p_{1}\right]=\kappa_{2} \lambda_{2} E\left[E\left[\theta \mid y, p_{1}\right] \mid y, p_{1}\right]+\kappa_{2} \mu_{2} E\left[\theta \mid y, p_{1}\right]=y_{2}$ and $\operatorname{Var}\left[p_{2} \mid y, p_{1}\right]=\left(\kappa_{2} \mu_{2}\right)^{2} \operatorname{Var}\left[\theta \mid y, p_{1}\right]+\kappa_{2}^{2} / \gamma=\left(\kappa_{2}^{2} / \gamma\right)\left(\alpha+\mu_{1}^{2} \gamma+\mu_{2}^{2} \gamma\right)\left(\alpha+\mu_{1}^{2} \gamma\right)^{-1}$. Similarly, private beliefs of informed traders follow the normal distribution with $E\left[p_{2} \mid y, p_{1}, x_{i 1}\right]=\kappa_{2} \lambda_{2} E\left[\theta \mid y, p_{1}\right]+$ $\kappa_{2} \mu_{2} E\left[\theta \mid y, p_{1}, x_{i 1}\right]$ and $\operatorname{Var}\left[p_{2} \mid y, p_{1}, x_{i 1}\right]=\left(\kappa_{2} \mu_{2}\right)^{2} \operatorname{Var}\left[\theta \mid y, p_{1}, x_{i 1}\right]+\kappa_{2}^{2} / \gamma=\left(\kappa_{2}^{2} / \gamma\right)\left(\alpha+\beta+\mu_{1}^{2} \gamma+\right.$ $\left.\mu_{2}^{2} \gamma\right)\left(\alpha+\beta+\mu_{1}^{2} \gamma\right)^{-1}$. Substituting these expressions into the market equilibrium condition for period 1 yields

$$
\int_{0}^{\phi} \frac{E\left[p_{2} \mid y, p_{1}, x_{i 1}\right]-p_{1}}{\tau^{-1} \operatorname{Var}\left[p_{2} \mid y, p_{1}, x_{i 1}\right]} d i+(1-\phi) \frac{E\left[p_{2} \mid y, p_{1}\right]-p_{1}}{\tau^{-1} \operatorname{Var}\left[p_{2} \mid y, p_{1}\right]}=s_{1}
$$

By solving for $p_{1}$, we obtain

$$
p_{1}=\frac{\phi \tau \frac{\kappa_{2} \lambda_{2}\left(\alpha-\mu_{1} \gamma \lambda_{1}\right) y \frac{\alpha+\beta+\mu_{1}^{2} \gamma}{\alpha+\mu_{1}^{2} \gamma}+\kappa_{2} \mu_{2}\left[\left(\alpha-\mu_{1} \gamma \lambda_{1}\right) y+\beta \theta\right]}{\frac{\kappa_{2}^{2}}{\gamma}\left(\alpha+\beta+\mu_{1}^{2} \gamma+\mu_{2}^{2} \gamma\right)}+(1-\phi) \tau \frac{\left(\alpha-\mu_{1} \gamma \lambda_{1}\right) y}{\frac{\kappa_{2}^{2}}{\gamma}\left(\alpha+\mu_{1}^{2} \gamma+\mu_{2}^{2} \gamma\right)}-s_{1}}{\phi \tau \frac{-\kappa_{2} \lambda_{2} \frac{\alpha+\beta+\mu_{1}^{2} \gamma}{\alpha+\mu_{1}^{2} \gamma} \frac{\mu_{1} \gamma}{\kappa_{1}}-\kappa_{2} \mu_{2} \frac{\mu_{1} \gamma}{\kappa_{1}}+\alpha+\beta+\mu_{1}^{2} \gamma}{\frac{\kappa_{2}^{2}}{\gamma}\left(\alpha+\beta+\mu_{1}^{2} \gamma+\mu_{2}^{2} \gamma\right)}+(1-\phi) \tau \frac{-\frac{\mu_{1} \gamma}{\kappa_{1}}+\alpha+\mu_{1}^{2} \gamma}{\frac{\kappa_{2}^{2}}{\gamma}\left(\alpha+\mu_{1}^{2} \gamma+\mu_{2}^{2} \gamma\right)}} .
$$

By matching coefficients with $p_{1}=\kappa_{1} \lambda_{1} y+\kappa_{1} \mu_{1} \theta-\kappa_{1} s_{1}$, we get

$$
\begin{aligned}
\frac{1}{\kappa_{1}} & =\phi \tau \frac{-\kappa_{2} \lambda_{2} \frac{\alpha+\beta+\mu_{1}^{2} \gamma}{\alpha+\mu_{1}^{2} \gamma} \frac{\mu_{1} \gamma}{\kappa_{1}}-\kappa_{2} \mu_{2} \frac{\mu_{1} \gamma}{\kappa_{1}}+\alpha+\beta+\mu_{1}^{2} \gamma}{\frac{\kappa_{2}^{2}}{\gamma}\left(\alpha+\beta+\mu_{1}^{2} \gamma+\mu_{2}^{2} \gamma\right)}+(1-\phi) \tau \frac{-\frac{\mu_{1} \gamma}{\kappa_{1}}+\alpha+\mu_{1}^{2} \gamma}{\frac{\kappa_{2}^{2}}{\gamma}\left(\alpha+\mu_{1}^{2} \gamma+\mu_{2}^{2} \gamma\right)}, \\
\lambda_{1} & =\phi \tau \frac{\kappa_{2} \lambda_{2}\left[\alpha-\mu_{1} \gamma \lambda_{1}\right] \frac{\alpha+\beta+\mu_{1}^{2} \gamma}{\alpha+\mu_{1}^{2} \gamma}+\kappa_{2} \mu_{2}\left[\alpha-\mu_{1} \gamma \lambda_{1}\right]}{\frac{\kappa_{2}^{2}}{\gamma}\left(\alpha+\beta+\mu_{1}^{2} \gamma+\mu_{2}^{2} \gamma\right)}+(1-\phi) \tau \frac{\left(\alpha-\mu_{1} \gamma \lambda_{1}\right)}{\frac{\kappa_{2}^{2}}{\gamma}\left(\alpha+\mu_{1}^{2} \gamma+\mu_{2}^{2} \gamma\right)}, \\
\mu_{1} & =\frac{\phi \tau \kappa_{2} \mu_{2} \beta}{\frac{\kappa_{2}^{2}}{\gamma}\left(\alpha+\beta+\mu_{1}^{2} \gamma+\mu_{2}^{2} \gamma\right)} .
\end{aligned}
$$

It is evident that a linear NREE is determined by solving the system of equations for the (endogenous) coefficients (11)-(17). It is important to observe that equations (11)-(13) alone cannot pin down the values of $\kappa_{2}, \lambda_{2}$, and $\mu_{2}$ because (12) contains the unknown $\mu_{1}$. Thus, our first task is to determine $\mu_{1}$. Equation (17) implies $\mu_{1} \kappa_{2}\left(\alpha+\beta+\mu_{1}^{2} \gamma+\mu_{2}^{2} \gamma\right)=\phi \tau \mu_{2} \beta \gamma$, which reduces to

$$
\gamma \mu_{1}^{3}-\gamma A \mu_{1}^{2}+(\beta+B) \mu_{1}-A(B+\phi \beta)=0
$$

where $A \equiv\left[1+\tau^{2} \phi \beta \gamma\right]^{-1} \tau(\phi \tau \beta)^{2} \gamma$ and $B \equiv \alpha+(\phi \tau \beta)^{2} \gamma$. Thus, there are potentially three linear equilibria, each of which is characterized by a distinct value of $\mu_{1}$. Given $\mu_{1}$, the values of $\kappa_{2}$ and 
$\lambda_{2}$ are determined by (11) and (12). Given these values, (15) determines $\kappa_{1}$ and (16) determines $\lambda_{1}$. In other words, the number of NREE is determined by the number of solutions to (18).

Proposition 4 There is a unique linear NREE.

Proof. Let $f\left(\mu_{1}\right) \equiv \gamma \mu_{1}^{3}-\gamma A \mu_{1}^{2}+(B+\beta) \mu_{1}-A B-A \phi \beta$, so $f^{\prime}\left(\mu_{1}\right)=3 \gamma \mu_{1}^{2}-2 \gamma A \mu_{1}+B+\beta$. Equation $f^{\prime}\left(\mu_{1}\right)=0$ has two distinct roots if and only if its determinant is positive: $(2 \gamma A)^{2}-$ $4(3 \gamma)(B+\beta)>0$, or, $\gamma\left[(\phi \tau \beta)^{2} \tau \gamma\right]^{2}>3\left[\alpha+(\phi \tau \beta)^{2} \gamma+\beta\right][1+(\tau \phi \beta) \tau \gamma]^{2}$, which further reduces to

$$
0>3[\alpha+\beta][1+(\tau \phi \beta) \tau \gamma]^{2}+3(\phi \tau \beta)^{2} \gamma[1+2(\tau \phi \beta) \tau \gamma]+2 \gamma\left[(\phi \tau \beta)^{2} \tau \gamma\right]^{2} .
$$

Clearly, this condition is violated. This establishes that $f\left(\mu_{1}\right)$ is monotonic, which implies that there is only one $\mu_{1}$ that solves $f\left(\mu_{1}\right)=0$.

This result is important. Recent literature has explored the issue of multiple equilibria in noisy rational expectations models of the stock market. Cespa (2002), for example, found that combining a short horizon with serially correlated noise trading leads to multiple linear equilibria. Cespa and Vives (2007) generalized Cespa (2002) to clarify the relationship between the serial correlation of noise trading and the likelihood of equilibrium multiplicity. Our framework is a special case of Cespa (2002) and Cespa and Vives (2007) in the sense that noise trading is not serially correlated, as is the case in AMS.

Under symmetric (but differential) information, the absence of serial correlation leads to a unique equilibrium, as has been shown in the literature. However, under asymmetric information, whether uniqueness is obtained is not trivial. In this context, notable studies are those of Gennotte and Leland (1990), and more recently, Barlevy and Veronesi (2003), who showed in their static models that there are multiple NREE under asymmetric information. This occurs because uninformed traders (i.e., market makers) are not certain whether they are trading against noise traders or informed traders, which constitutes the adverse selection problem in financial markets. The importance of Proposition 4 is that beauty contests under asymmetric information in a dynamic setting do not generate equilibrium multiplicity. 


\subsection{Information and Volatility}

In this section, we present numerical examples. The benchmark parameter values are given in Table 1 below:

Table 1: Example 1

\begin{tabular}{|l|l|l|l|l|}
\hline$y=1$ & $\tau=1$ & $\alpha=1$ & $\beta=1$ & $\gamma=1$ \\
\hline
\end{tabular}

To highlight the information content of the asset prices, we write the price equations in terms of $y$ and $\theta$ in addition to $y_{2}$ and $\theta$. To do this, we substitute $y_{2}$ and the price conjecture about $p_{1}$ into the price conjecture about $p_{2}$ to obtain

$$
p_{2}=\frac{\kappa_{2} \lambda_{2} \alpha}{\alpha+\mu_{1}^{2} \gamma} y+\left(\frac{\kappa_{2} \lambda_{2} \mu_{1}^{2} \gamma}{\alpha+\mu_{1}^{2} \gamma}+\kappa_{2} \mu_{2}\right) \theta-\frac{\kappa_{2} \lambda_{2} \mu_{1} \gamma}{\alpha+\mu_{1}^{2} \gamma} s_{1}-\kappa_{2} s_{2}
$$

From this equation, we obtain the following measure of asset price volatility:

$$
\operatorname{Var}\left[p_{2}-p_{1}\right]=\left(\frac{\kappa_{2} \lambda_{2} \mu_{1}^{2} \gamma}{\alpha+\mu_{1}^{2} \gamma}+\kappa_{2} \mu_{2}-\kappa_{1} \mu_{1}\right)^{2} \alpha+\left(\frac{\kappa_{2} \lambda_{2} \mu_{1} \gamma}{\alpha+\mu_{1}^{2} \gamma}-\kappa_{1}\right)^{2} \gamma+\kappa_{2}^{2} \gamma
$$

We start with the benchmark economy, in which $\phi=1$. This case corresponds to the AMS model. In this case, we obtain the following the equilibrium price equations:

$$
\begin{aligned}
p_{1} & =\underbrace{0.5812}_{\text {Reliance on public information }} y+\underbrace{0.4188}_{\text {Information content }} \theta-\underbrace{0.8376}_{1 / \text { Market liquidity }} s_{1}, \\
p_{2} & =\underbrace{0.3846}_{\text {Reliance on public information }} y_{2}+0.6154 \theta-\underbrace{0.6154}_{\text {I/Market liquidity }} s_{2},
\end{aligned}
$$

Two results are worth mentioning. First, this example replicates AMS's main result that, as time passes, asset prices contain more information about fundamentals in the sense that the price equation for period 2 puts more weight on $\theta .{ }^{11}$ This can easily be verified by comparing 0.6923 with 0.4188. Second, the period-2 price has a smaller weight on the public belief about the fundamental, $y_{2}$ : it decreases from 0.5812 to 0.3846 .

Now we consider the impact of asymmetric information. Figure 2 shows the information content of the price (panel a), the price's reliance on public information (panel b), market liquidity (panel

\footnotetext{
${ }^{11}$ This result was also obtained in He and Wang (1995), who considered a multiperiod noisy rational expectations model with long-horizon investors.
} 
c), and price volatility (panel d) for each level of $\phi$. In this example, the information content is monotonically increasing in $\phi$, and it is increasing over time for each $\phi>0$. The price's reliance on public information is monotonically decreasing in $\phi$ because the information content is increasing. For each $\phi>0$, the price's reliance on public information decreases over time because the price reflects more information over time.

Market liquidity is monotonically increasing in $\phi$ in each period, and increases over time for each $\phi>0$. This suggests that, on average, traders have demand curves that are flatter in period 2 than in period 1 because the adverse selection problem diminishes over time. As a result, price volatility $\operatorname{Var}\left[p_{2}-p_{1}\right]$ decreases as $\phi$ increases.

Consider another example. The parameter values are given in Table 2 below:

Table 2: Example 2

\begin{tabular}{|l|l|l|l|l|}
\hline$y=1$ & $\tau=1$ & $\alpha=2$ & $\beta=3$ & $\gamma=1$ \\
\hline
\end{tabular}

In this example, private information is more informative than public information $(\beta>\alpha)$, and noise trading is more volatile than public information $(\alpha>\gamma)$.

The results are shown in Figure 3. In this example, the information content (panel a) is monotonically increasing in $\phi$, and increases over time for each $\phi>0$. The price's reliance on public information (panel b) is monotonically decreasing in $\phi$ because the information content is increasing. However, this reliance increases over time for large values of $\phi$. The signals in Example 2 are more informative than those in Example 1, whereas the variance of noise trading is the same in both examples. For small values of $\phi$, the price's reliance on public information decreases over time because the price reflects more information over time. However, for large values of $\phi$, as $\phi$ increases, the information content of $p_{1}$ increases, which increases the precision of the public belief in period 2. Since the public belief in period 2 is sufficiently informative, the price's reliance on public information increases over time, as does the information content of the price.

Market liquidity (panel c) is nonmonotonic in $\phi$ in each period, as the static model suggests (Proposition 3). As a result, there exists a threshold level of $\phi$ such that market liquidity decreases over time for any $\phi$ below the threshold. In other words, $\kappa_{1}^{-1}=\phi \tau / \operatorname{Var}\left[p_{2} \mid \Omega_{i 1}\right]+(1-$ 
$\phi) \tau / \operatorname{Var}\left[p_{2} \mid \Omega_{1}\right]>\phi \tau / \operatorname{Var}\left[\theta \mid \Omega_{i 2}\right]+(1-\phi) \tau / \operatorname{Var}\left[\theta \mid \Omega_{2}\right]=\kappa_{2}^{-1}$ holds.

A possible interpretation is as follows. For small values of $\phi$, the asset demand curves in period 2 have, on average, steeper slopes than the demand curves in period 1. Remember that the informed traders have a greater advantage in this example $(\beta>\alpha)$. This makes the adverse selection problem more serious, and it stimulates the speculative demand of informed traders. For small values of $\phi$, little information is integrated into the price in period 1 . Thus, in period 2 , the economy is similar to the static economy because the public belief is similar to the prior belief. Less information in $p_{2}$ implies that $p_{2}$ is not strongly influenced by variations in private information. This implies a low variance of $p_{2}$. This induces an increase in market making positions in period 1. As a result, the adverse selection problem is less severe in period 1 than in period 2 .

Interestingly, price volatility $\operatorname{Var}\left[p_{2}-p_{1}\right]$ is nonmonotonic in $\phi$ in this example. In Example 1, price volatility monotonically increases as the proportion of uninformed traders increases. This is because the adverse selection problem deteriorates and fewer market making positions are taken. In Example 2, when the proportion of uninformed traders exceeds a threshold level, any further increase in the proportion of uninformed traders stabilizes the market.

Consider the next parameter values given in Table 3 below:

Table 3: Example 3

$$
\begin{array}{|l|l|l|l|l|}
\hline y=1 & \tau=1 & \alpha=2 & \beta=0.5 & \gamma=1 \\
\hline
\end{array}
$$

In this example, private information is noisy. As shown in Figure 4, information content (panel a) is increasing in $\phi$, and increases over time. The price's reliance on public information is decreasing in $\phi$, and decreases over time. Interestingly, market liquidity (panel c) decreases over time, and price volatility (panel d) increases as $\phi$ increases because of the noisy private information. That is, imprecise information is destabilizing. 


\subsection{Equilibrium Behavior of Traders}

Let us now consider the equilibrium behavior of each trader. Given the equilibrium coefficients, we can derive (after tedious calculations) the asset demand function for each trader as follows:

$$
\begin{aligned}
X_{i 1} & =\frac{\left[1+\frac{\left(1-\kappa_{2} \mu_{2}\right) \beta}{\alpha+\mu_{1}^{2} \gamma}\right] \tau \alpha\left(y-p_{1}\right)+\left[1+\frac{\left(1-\kappa_{2} \mu_{2}\right) \beta}{\alpha+\mu_{1}^{2} \gamma}\right] \tau \mu_{1}^{2} \gamma\left(z_{1}-p_{1}\right)+\kappa_{2} \mu_{2} \tau \beta\left(x_{i 1}-p_{1}\right)}{\frac{\kappa_{2}^{2}}{\gamma}\left(\alpha+\beta+\mu_{1}^{2} \gamma+\mu_{2}^{2} \gamma\right)} \\
X_{1}^{U} & =\frac{\tau \alpha\left(y-p_{1}\right)+\tau \mu_{1}^{2} \gamma\left(z_{1}-p_{1}\right)}{\frac{\kappa_{2}^{2}}{\gamma}\left(\alpha+\mu_{1}^{2} \gamma+\mu_{2}^{2} \gamma\right)} \\
X_{i 2} & =\tau \alpha_{2}\left(y_{2}-p_{2}\right)+\tau \mu_{2}^{2} \gamma\left(z_{2}-p_{2}\right)+\tau \beta\left(x_{i 2}-p_{2}\right) \\
X_{2}^{U} & =\tau \alpha_{2}\left(y_{2}-p_{2}\right)+\tau \mu_{2}^{2} \gamma\left(z_{2}-p_{2}\right)
\end{aligned}
$$

According to these demand functions, the uninformed trader's demand is decomposed into two terms. The first term captures the intensity of trade that responds to variations in public expectations just prior to trade (i.e., prior to receiving the price signal). Since the coefficient is positive, the trader's position is positive if and only if the price is below public expectations about the fundamentals. The second term captures the intensity of trade that responds to the difference between the public signal about the fundamental $\left(z_{2}\right)$ and the price. It is interesting to observe that, since $z_{2}-p_{2}=\left(\lambda_{2} / \mu_{2}\right)\left(p_{2}-y_{2}\right)$, the effect is such that if the price is above the public belief, the position must be positive, because higher prices are likely to imply strong fundamentals.

Consider the parameter values given in Table 2 . For $\phi=0.9$, we have

$$
\begin{aligned}
& X_{i 1}=2.6848\left(y-p_{1}\right)+5.0179\left(z_{1}-p_{1}\right)+2.1482\left(x_{i 1}-p_{1}\right), \\
& X_{1}^{U}=2.7739\left(y-p_{1}\right)+5.1845\left(z_{1}-p_{1}\right), \\
& X_{i 2}=5.7380\left(y_{2}-p_{2}\right)+7.2900\left(z_{2}-p_{2}\right)+3.0000\left(x_{i 2}-p_{2}\right), \\
& X_{2}^{U}=5.7380\left(y_{2}-p_{2}\right)+2.2900\left(z_{2}-p_{2}\right) .
\end{aligned}
$$

To study the impact of asymmetric information, consider the case with $\phi=0.2$. The asset demand 
functions are:

$$
\begin{aligned}
& X_{i 1}=2.5841\left(y-p_{1}\right)+0.0200\left(z_{1}-p_{1}\right)+0.6227\left(x_{i 1}-p_{1}\right), \\
& X_{1}^{U}=2.9118\left(y-p_{1}\right)+0.0226\left(z_{1}-p_{1}\right), \\
& X_{i 2}=2.0155\left(y_{2}-p_{2}\right)+0.3600\left(z_{2}-p_{2}\right)+3.0000\left(x_{i 2}-p_{2}\right), \\
& X_{2}^{U}=2.0155\left(y_{2}-p_{2}\right)+0.36\left(z_{2}-p_{2}\right) .
\end{aligned}
$$

Since there is only a small proportion of informed traders, the price signal is not very informative, so traders do not react strongly to the price signal $z_{t}$ in any period. This can be verified by comparing the coefficients on $\left(z_{1}-p_{1}\right)$ or those on $\left(z_{2}-p_{2}\right)$. For example, the uninformed trader's coefficient on $\left(z_{1}-p_{1}\right)$ is 5.1845 under $\phi=0.9$, and is 0.0226 under $\phi=0.2$.

In addition, traders in period 2 react less to the public belief under $\phi=0.2$ because the public belief contains less information. In fact, the coefficient on $\left(y_{2}-p_{2}\right)$ is 5.7380 under $\phi=0.9$, whereas it is 2.0155 under $\phi=0.2$. Even more interesting is the reaction to the public belief over time. It is easy to see that the coefficient on $\left(y_{t}-p_{t}\right)$ increases over time under $\phi=0.9$, but decreases over time under $\phi=0.2$. This implies that, over time, more market making positions are taken under $\phi=0.9$, whereas fewer are taken under $\phi=0.2$. As a result, market liquidity increases over time under $\phi=0.9$ but decreases over time under $\phi=0.2$, as shown in panel c of Figure 3 .

Interestingly, informed traders in period 1 do not react strongly to their private information under asymmetric information. In fact, the coefficient on $\left(x_{i 1}-p_{1}\right)$ is 2.1482 under $\phi=0.9$, whereas it is 0.6227 under $\phi=0.2$. In period 2, both coefficients are 3.0000. In other words, under asymmetric information, fewer speculative positions are taken by informed traders only in period 1 .

This may seem couterintuitive because speculative demand should depend primarily on risk tolerance and the precision of private information. In fact, (21) indicates that the coefficient on $\left(x_{i 2}-p_{2}\right)$ is $\tau \beta$ and is independent of $\phi$. However, (20) indicates that in period 1 , the extent of the speculation is influenced by $\kappa_{2} \mu_{2}$, the information content of the price in period 2 . This reflects the fact that, because of beauty contests, informed traders' decisions necessarily involve dynamic reasoning. In particular, even though the informed trader receives precise private information in 
period 1 , he or she cannot take a bold speculative position if the information content of the price in the next period is low. This is because his or her information advantage shrinks when the adverse selection problem is severe.

\section{$5 \quad$ Market Orders}

In the preceding sections, we maintained the assumption that all rational traders submit their demand schedules to the market. This type of market microstructure is referred to as (generalized) limit orders. Another major market microstructure often studied in the literature is market orders, under which traders submit quantities of assets demanded. The purpose of this section is to briefly study market orders to highlight the role of market microstructure.

The key assumption in this section is that the informed agents and noise traders move first (Chamley, 2004; Vives, 2008). There are two important implications. One is that the informed agents cannot observe the current asset price: there is no learning from the current price. The other is that uninformed traders necessarily act as market makers. Thus, information is revealed more slowly under market orders than under limit orders. The key question in this section is whether the results obtained under limit orders are maintained.

Suppose that agents form a common conjecture about the period 1 price such that $p_{1}=\kappa_{1}\left(\lambda_{1} y+\right.$ $\left.\mu_{1} \theta-s_{1}\right)$, from which we obtain $z_{1} \equiv\left(p_{1}-\kappa_{1} \lambda_{1} y\right) / \kappa_{1} \mu_{1}=\theta-s_{1} / \mu_{1}$. The price signal $z_{1}$ is normally distributed with mean $\theta$ and precision $\mu_{1}^{2} \gamma$. In period 1 , after observing $p_{1}$, uninformed agents' beliefs about $\theta$ are updated to the normal distribution with $E\left[\theta \mid y, p_{1}\right]=\left(\alpha+\mu_{1}^{2} \gamma\right)^{-1}\left[\left(\alpha-\mu_{1} \gamma \lambda_{1}\right) y+\right.$ $\left.\left(\mu_{1} \gamma / \kappa_{1}\right) p_{1}\right] \equiv y_{2}$ and $\operatorname{Var}\left[\theta \mid y, p_{1}\right]=\left(\alpha+\mu_{1}^{2} \gamma\right)^{-1} \equiv 1 / \alpha_{2}$. Informed traders observe private signals. Their posterior beliefs follow the normal distribution with $E\left[\theta \mid y, x_{i 1}\right]=(\alpha+\beta)^{-1}\left(\alpha y+\beta x_{i 1}\right)$ and $\operatorname{Var}\left[\theta \mid y, x_{i 1}\right]=(\alpha+\beta)^{-1}$.

Similarly, suppose that agents form a common price conjecture about the period-2 price such that $p_{2}=\kappa_{2}\left(\lambda_{2} y_{2}+\mu_{2} \theta-s_{2}\right)$, from which we get $z_{2} \equiv\left(p_{2}-\kappa_{2} \lambda_{2} y_{2}\right) / \kappa_{2} \mu_{2}=\theta-s_{2} / \mu_{2}$. The random variable $z_{2}$ is normally distributed with mean $\theta$ and precision $\mu_{2}^{2} \gamma$, and is informationally equivalent to the price. Thus, in period 2, after observing the price, uninformed agents' beliefs 
about $\theta$ are updated to the normal distribution with $E\left[\theta \mid y, p_{1}, p_{2}\right]=\left(\alpha_{2}+\mu_{2}^{2} \gamma\right)^{-1}\left[\left(\alpha_{2}-\mu_{2} \gamma \lambda_{2}\right) y_{2}+\right.$ $\left.\left(\mu_{2} \gamma / \kappa_{2}\right) p_{2}\right]$ and $\operatorname{Var}\left[\theta \mid y, p_{1}, p_{2}\right]=\left(\alpha_{2}+\mu_{2}^{2} \gamma\right)^{-1}$. The posterior beliefs of informed traders, who cannot observe $p_{2}$, follow the normal distribution with $E\left[\theta \mid y, p_{1}, x_{i 2}\right]=\left(\alpha_{2}+\beta\right)^{-1}\left(\alpha_{2} y_{2}+\beta x_{i 2}\right)$ and $\operatorname{Var}\left[\theta \mid y, p_{1}, x_{i 2}\right]=\left(\alpha_{2}+\beta\right)^{-1}$.

Now consider the determination of equilibrium in period 2. Market equilibrium requires

$$
\int_{0}^{\phi} \frac{E\left[\theta \mid y, p_{1}, x_{i 2}\right]-p_{2}}{\tau^{-1} \operatorname{Var}\left[\theta \mid y, p_{1}, x_{i 2}\right]} d i+(1-\phi) \frac{E\left[\theta \mid y, p_{1}, p_{2}\right]-p_{2}}{\tau^{-1} \operatorname{Var}\left[\theta \mid y, p_{1}, p_{2}\right]}=s_{2}
$$

We solve this for $p_{2}$ to obtain

$$
p_{2}=\frac{\left[\tau \alpha_{2}-\left(1-\phi_{2}\right) \tau \mu_{2} \gamma \lambda_{2}\right] y_{2}+\phi \tau \beta \theta-s_{2}}{\tau \alpha_{2}+\phi \tau \beta+(1-\phi) \tau \mu_{2} \gamma\left[\mu_{2}-1 / \kappa_{2}\right]} .
$$

Rational expectations require that $\kappa_{2}^{-1}=\tau \alpha_{2}+\phi \tau \beta_{2}+(1-\phi) \tau \mu_{2} \gamma\left[\mu_{2}-1 / \kappa_{2}\right], \lambda_{2}=\tau \alpha_{2}-(1-$ $\phi) \tau \mu_{2} \gamma \lambda_{2}, \mu_{2}=\phi \tau \beta$, from which we obtain

$$
\kappa_{2}=\frac{1+(1-\phi) \tau(\phi \tau \beta) \gamma}{\tau\left(\alpha+\mu_{1}^{2} \gamma\right)+[1+(1-\phi) \tau(\phi \tau \beta) \gamma] \phi \tau \beta}, \lambda_{2}=\frac{\tau\left(\alpha+\mu_{1}^{2} \gamma\right)}{1+(1-\phi) \tau(\phi \tau \beta) \gamma}, \mu_{2}=\phi \tau \beta .
$$

It is evident that $\kappa_{2} \lambda_{2}+\kappa_{2} \mu_{2}=1$.

Consider stock market equilibrium in period 1. With the price conjecture, uninformed agents' beliefs about $p_{2}$ follow the normal distribution with $E\left[p_{2} \mid y, p_{1}\right]=y_{2}$ and $\operatorname{Var}\left[p_{2} \mid y, p_{1}\right]=\left(\kappa_{2} \mu_{2}\right)^{2} \operatorname{Var}\left[\theta \mid y, p_{1}\right]+$ $\kappa_{2}^{2} / \gamma=\left(\kappa_{2} \mu_{2}\right)^{2} /\left(\alpha+\mu_{1}^{2} \gamma\right)+\kappa_{2}^{2} / \gamma$. The computation of $E\left[p_{2} \mid y, x_{i 1}\right]$ and $\operatorname{Var}\left[p_{2} \mid y, x_{i 1}\right]$ is more complicated because informed traders cannot observe $p_{1}$. Substituting $y_{2}$ and the price conjecture about $p_{1}$ into the price conjecture about $p_{2}$ yields (19), from which it is easy to show that the private beliefs of informed traders are the normal with

$$
\begin{aligned}
E\left[p_{2} \mid y, x_{i 1}\right] & =\frac{\kappa_{2} \lambda_{2} \alpha}{\alpha+\mu_{1}^{2} \gamma} y+\left(\frac{\kappa_{2} \lambda_{2} \mu_{1}^{2} \gamma}{\alpha+\mu_{1}^{2} \gamma}+\kappa_{2} \mu_{2}\right) E\left[\theta \mid y, x_{i 1}\right] \\
\operatorname{Var}\left[p_{2} \mid y, x_{i 1}\right] & =\left(\frac{\kappa_{2} \lambda_{2} \mu_{1}^{2} \gamma}{\alpha+\mu_{1}^{2} \gamma}+\kappa_{2} \mu_{2}\right)^{2} \operatorname{Var}\left[\theta \mid y, x_{i 1}\right]+\left(\frac{\kappa_{2} \lambda_{2} \mu_{1} \gamma}{\alpha+\mu_{1}^{2} \gamma}\right)^{2} \frac{1}{\gamma}+\kappa_{2}^{2} \frac{1}{\gamma} .
\end{aligned}
$$

Thus, the market equilibrium condition for period 1 is

$$
\int_{0}^{\phi} \frac{E\left[p_{2} \mid y, x_{i 1}\right]-p_{1}}{\tau^{-1} \operatorname{Var}\left[p_{2} \mid y, x_{i 1}\right]} d i+(1-\phi) \frac{E\left[p_{2} \mid y, p_{1}\right]-p_{1}}{\tau^{-1} \operatorname{Var}\left[p_{2} \mid y, p_{1}\right]}=s_{1}
$$


Solving this for $p_{1}$ yields

$$
p_{1}=\frac{\phi \tau \frac{\frac{\kappa_{2} \lambda_{2} \alpha}{\alpha+\mu_{1}^{2} \gamma} y+\left(\frac{\kappa_{2} \lambda_{2} \mu_{1}^{2} \gamma}{\alpha+\mu_{1}^{2} \gamma}+\kappa_{2} \mu_{2}\right) \frac{\alpha y+\beta \theta}{\alpha+\beta}}{\left(\frac{\kappa_{2} \lambda_{2} \mu_{1}^{2} \gamma}{\alpha+\mu_{1}^{2} \gamma}+\kappa_{2} \mu_{2}\right)^{2}(\alpha+\beta)^{-1}+\left(\frac{\kappa_{2} \lambda_{2} \mu_{1} \gamma}{\alpha+\mu_{1}^{2} \gamma}\right)^{2} \frac{1}{\gamma}+\frac{\kappa_{2}^{2}}{\gamma}}+\frac{(1-\phi) \tau\left(\alpha-\mu_{1} \gamma \lambda_{1}\right) y}{\left(\kappa_{2} \mu_{2}\right)^{2}+\left(\alpha+\mu_{1}^{2} \gamma\right) \frac{\kappa_{2}^{2}}{\gamma}}-s_{1}}{\frac{\phi \tau}{\left(\frac{\kappa_{2} \lambda_{2} \mu_{1}^{2} \gamma}{\alpha+\mu_{1}^{2} \gamma}+\kappa_{2} \mu_{2}\right)^{2}(\alpha+\beta)^{-1}+\left(\frac{\kappa_{2} \lambda_{2} \mu_{1} \gamma}{\alpha+\mu_{1}^{2} \gamma}\right)^{2} \frac{1}{\gamma}+\frac{\kappa_{2}^{2}}{\gamma}}+\frac{(1-\phi) \tau\left[1-\frac{\mu_{1}}{\kappa_{1}} \gamma\left(\alpha+\mu_{1}^{2} \gamma\right)^{-1}\right]}{\frac{\left(\kappa_{2} \mu_{2}\right)^{2}}{\alpha+\mu_{1}^{2} \gamma}+\frac{\kappa_{2}^{2}}{\gamma}}} .
$$

By matching coefficients with $p_{1}=\kappa_{1}\left(\lambda_{1} y+\mu_{1} \theta-s_{1}\right)$, we obtain

$$
\begin{aligned}
\kappa_{1}^{-1} & =\frac{\phi \tau}{\left(\frac{\kappa_{2} \lambda_{2} \mu_{1}^{2} \gamma}{\alpha+\mu_{1}^{2} \gamma}+\kappa_{2} \mu_{2}\right)^{2}(\alpha+\beta)^{-1}+\left(\frac{\kappa_{2} \lambda_{2} \mu_{1} \gamma}{\alpha+\mu_{1}^{2} \gamma}\right)^{2} \frac{1}{\gamma}+\frac{\kappa_{2}^{2}}{\gamma}}+\frac{(1-\phi) \tau\left[1-\frac{\frac{\mu_{1}}{\kappa_{1}} \gamma}{\alpha+\mu_{1}^{2} \gamma}\right]}{\frac{\left(\kappa_{2} \mu_{2}\right)^{2}}{\alpha+\mu_{1}^{2} \gamma}+\frac{\kappa_{2}^{2}}{\gamma}}, \\
\lambda_{1} & =\phi \tau \frac{\frac{\kappa_{2} \lambda_{2} \alpha}{\alpha+\mu_{1}^{2} \gamma}\left[1+\frac{\mu_{1}^{2} \gamma}{\alpha+\beta}\right]+\kappa_{2} \mu_{2} \frac{\alpha}{\alpha+\beta}}{\left(\frac{\kappa_{2} \lambda_{2} \mu_{1}^{2} \gamma}{\alpha+\mu_{1}^{2} \gamma}+\kappa_{2} \mu_{2}\right)^{2}(\alpha+\beta)^{-1}+\left(\frac{\kappa_{2} \lambda_{2} \mu_{1} \gamma}{\alpha+\mu_{1}^{2} \gamma}\right)^{2} \frac{1}{\gamma}+\frac{\kappa_{2}^{2}}{\gamma}}+\frac{(1-\phi) \tau\left(\alpha-\mu_{1} \gamma \lambda_{1}\right)}{\left(\kappa_{2} \mu_{2}\right)^{2}+\left(\alpha+\mu_{1}^{2} \gamma\right) \frac{\kappa_{2}^{2}}{\gamma}} \\
\mu_{1} & =\frac{\phi \tau\left(\frac{\kappa_{2} \lambda_{2} \mu_{1}^{2} \gamma}{\alpha+\mu_{1}^{2} \gamma}+\kappa_{2} \mu_{2}\right) \frac{\beta}{\alpha+\beta}}{\left(\frac{\kappa_{2} \lambda_{2} \mu_{1}^{2} \gamma}{\alpha+\mu_{1}^{2} \gamma}+\kappa_{2} \mu_{2}\right)^{2}(\alpha+\beta)^{-1}+\left(\frac{\kappa_{2} \lambda_{2} \mu_{1} \gamma}{\alpha+\mu_{1}^{2} \gamma}\right)^{2} \frac{1}{\gamma}+\frac{\kappa_{2}^{2}}{\gamma}}
\end{aligned}
$$

The solution to this system of undetermined coefficients determines a linear NREE.

Notice that substituting (22) into (25) yields a single nonlinear equation in terms of $\mu_{1}$. It is then easy, though tedious, to show that the equilibrium value of $\mu_{1}$ is determined as the solution to:

$$
\mu_{1}=\frac{\phi \tau \beta\left[\alpha+\mu_{1}^{2} \gamma+\Phi \phi \beta\right]\left[\mu_{1}^{2} \gamma+\Phi \phi \beta\right]}{\left(\mu_{1}^{2} \gamma+\Phi \phi \beta\right)^{2}+\frac{\alpha+\beta}{\gamma}\left[\left(\mu_{1} \gamma\right)^{2}+\Phi^{2} / \tau^{2}\right]},
$$

where $\Phi \equiv 1+(1-\phi) \tau(\phi \tau \beta) \gamma$. Once the value of $\mu_{1}$ is given, the other coefficients are easily derived from (22)-(25).

Equation (26) has at most five roots. Instead of trying to obtain a general result about uniqueness, we present some numerical examples to highlight the properties of the model. We use the parameter values in Tables 1 and 2. The results are summarized in Figures 5 and 6 . The dashed lines represent the results under limit orders. It is then easy to verify that the information content of the price under market orders is generally below that under limit orders. However, for many parameter values, price volatility under market orders is below that under limit orders. 


\section{Conclusion}

In this paper, we analyzed the role of asymmetric information in a dynamic financial market in which higher-order expectations matter. In a two-period economy, we have established that there is a unique linear equilibrium; beauty contests under asymmetric information do not introduce excess volatility driven by equilibrium multiplicity. This result contrasts with the findings of Cespa (2002) and Cespa and Vives (2007), who found multiple linear equilibria under symmetric information.

Keynes observed that "ignorance" in the stock market can be destabilizing. The analysis in this paper suggests that ignorance is not always destabilizing. In particular, it is possible for price volatility to decrease with the proportion of uninformed traders. Such a result occurs when the adverse selection problem is severe: uninformed traders feel safer as the proportion of informed traders decreases.

Abreu and Brunnermeier (2003) introduced the concept of an "awareness window" to compute the timing of a crash. In their model, a crash occurs when a sufficiently large number of traders are sure that enough traders are aware of mispricing. In their model, the time path of the asset price is exogenous. An important future research is to investigate the relationship between higher-order expectations and stock market crashes (as a unique equilibrium) by using a model in which asset prices, which convey information, are determined endogenously.

Using a static model, Romer (1993) showed that crashes are possible when traders are uncertain about the quality of other traders' information. Similarly, Avery and Zemsky (1998) showed that financial herding associated with mispricing is possible when there is uncertainty about the quality of traders' information. Extending our model to include uncertainty about the quality of information could shed light on the incidence of stock market crashes. 


\section{Appendix}

\section{A Proof of Proposition 1}

This proposition is proved by induction from $T$. Given (5) and noting that $p_{T+1}=\theta$, we solve the period- $T$ price equation for $p_{T}$ to obtain

$$
\begin{aligned}
p_{T} & =\frac{1}{\nu_{T}}\left\{\operatorname{Var}_{T}(\theta) \bar{E}_{T}(\theta)+M \operatorname{Var}_{i T}(\theta) E_{T}(\theta)-\frac{\operatorname{Var}_{T}(\theta) \operatorname{Var}_{i T}(\theta)}{\phi \tau} s_{T}\right\} \\
& =\sum_{k=0}^{1} \sum_{\tilde{s} \in \mathcal{S}(1, k)} \frac{M^{k} \operatorname{Var}_{\tilde{s}} \mathbf{E}_{\tilde{s}}(\theta)}{\nu_{T}}-\frac{\operatorname{Var}_{T}\left(p_{T+1}\right) \operatorname{Var}_{i T}\left(p_{T+1}\right)}{\nu_{T}} \frac{s_{T}}{\phi \tau},
\end{aligned}
$$

where $M \equiv(1-\phi) / \phi$. Suppose that (6) is satisfied in period $t=h+1 \leq T$ :

$$
p_{h+1}=\sum_{k=0}^{T-h} \sum_{\tilde{s} \in \mathcal{S}(T-h, k)} \frac{M^{k} \operatorname{Var}_{\tilde{s}} \mathbf{E}_{\tilde{s}}(\theta)}{\nu_{h+1} \nu_{h+2} \cdots \nu_{T}}-\frac{\operatorname{Var}_{h+1}\left(p_{h+2}\right) \operatorname{Var}_{i h+1}\left(p_{h+2}\right)}{\nu_{h+1}} \frac{s_{h+1}}{\phi \tau} .
$$

Then, by (5), it follows that in period $h$,

$$
\begin{aligned}
p_{h}= & \frac{1}{\nu_{h}}\left\{\operatorname{Var}_{h}\left(p_{h+1}\right) \bar{E}_{h}\left[\sum_{k=0}^{T-h} \sum_{\tilde{s} \in \mathcal{S}(T-h, k)} \frac{M^{k} \operatorname{Var}_{\tilde{s}} \mathbf{E}_{\tilde{s}}(\theta)}{\nu_{h+1} \nu_{h+2} \cdots \nu_{T}}-\frac{\operatorname{Var}_{h+1}\left(p_{h+2}\right) \operatorname{Var}_{i h+1}\left(p_{h+2}\right)}{\nu_{h+1}} \frac{s_{h+1}}{\phi \tau}\right]\right. \\
& +M \operatorname{Var}_{i h}\left(p_{h+1}\right) E_{h}\left[\sum_{k=0}^{T-h} \sum_{\tilde{s} \in \mathcal{S}(T-h, k)} \frac{M^{k} \operatorname{Var}_{\tilde{s}} \mathbf{E}_{\tilde{s}}(\theta)}{\nu_{h+1} \nu_{h+2} \cdots \nu_{T}}-\frac{\operatorname{Var}_{h+1}\left(p_{h+2}\right) \operatorname{Var}_{i h+1}\left(p_{h+2}\right)}{\nu_{h+1}} \frac{s_{h+1}}{\phi \tau}\right] \\
& \left.-\frac{\operatorname{Var}_{h}\left(p_{h+1}\right) \operatorname{Var}_{i h}\left(p_{h+1}\right)}{\phi \tau} s_{t}\right\} \\
= & \sum_{k=0}^{T-h+1} \sum_{\tilde{s} \in \mathcal{S}(T-h+1, k)} \frac{M^{k} \operatorname{Var}_{\tilde{s}} \mathbf{E}_{\tilde{s}}(\theta)}{\nu_{h} \nu_{h+1} \cdots \nu_{T}}-\frac{\operatorname{Var}_{h}\left(p_{h+1}\right) \operatorname{Var}_{i h}\left(p_{h+1}\right)}{\nu_{t}} \frac{s_{h}}{\phi \tau},
\end{aligned}
$$

where we have used $\bar{E}_{h}\left(s_{h+1}\right)=E_{h}\left(s_{h+1}\right)=0$.

\section{B Proof of Proposition 2}

Remember that public prior beliefs about the fundamentals are $\theta \sim N(y, 1 / \alpha)$, and that private information is $x_{i}=\theta+\varepsilon_{i}$, where $\varepsilon_{i} \sim N(0,1 / \beta)$, and $\varepsilon_{i}$ is independently and identically distributed across individuals. Suppose that agents form a common price conjecture such that $p=\kappa(\lambda y+$ $\mu \theta-s)$, where $\kappa, \lambda$, and $\mu$ are parameters to be determined. We rewrite the equation as $z \equiv$ 
$(p-\kappa \lambda y) / \kappa \mu=\theta-\frac{1}{\mu} s$. The random variable $z$ is normally distributed with mean $\theta$ and precision $\mu^{2} \gamma$. Thus, $z$ is a signal about $\theta$.

Thus, after observing the price, which is equivalent to observing $z$, public beliefs about $\theta$ are updated to the normal distribution with $E[\theta \mid y, p]=\left(\alpha+\mu^{2} \gamma\right)^{-1}\left(\alpha y+\mu^{2} \gamma z\right)=\left(\alpha+\mu^{2} \gamma\right)^{-1}[(\alpha-$ $\mu \gamma \lambda) y+(\mu \gamma / \kappa) p]$ and $\operatorname{Var}[\theta \mid y, p]=\left(\alpha+\mu^{2} \gamma\right)^{-1}$. Informed traders in addition observe private signals. After observing a private signal, informed trader $i$ updates his or her beliefs. The posterior beliefs follow the normal distribution with $E\left[\theta \mid y, p, x_{i}\right]=\left(\alpha+\beta+\mu^{2} \gamma\right)^{-1}\left[(\alpha-\mu \gamma \lambda) y+(\mu \gamma / \kappa) p+\beta x_{i}\right]$ and $\operatorname{Var}\left[\theta \mid y, p, x_{i}\right]=\left(\alpha+\beta+\mu^{2} \gamma\right)^{-1}$. Thus, the asset demands of informed and uninformed traders are $\tau\left[(\alpha-\mu \gamma \lambda) y+\frac{\mu \gamma}{\kappa} p+\beta x_{i}-\left(\alpha+\beta+\mu^{2} \gamma\right) p\right]$ and $\tau\left[(\alpha-\mu \gamma \lambda) y+\frac{\mu \gamma}{\kappa} p-\left(\alpha+\mu^{2} \gamma\right) p\right]$, respectively. Market equilibrium requires

$\phi \tau\left[(\alpha-\mu \gamma \lambda) y+\frac{\mu \gamma}{\kappa} p+\beta \theta-\left(\alpha+\beta+\mu^{2} \gamma\right) p\right]+(1-\phi) \tau\left[(\alpha-\mu \gamma \lambda) y+\frac{\mu \gamma}{\kappa} p-\left(\alpha+\mu^{2} \gamma\right) p\right]=s$

Solving this for $p$ gives

$$
p=\frac{\phi \tau \beta \theta+\tau(\alpha-\mu \gamma \lambda) y-s}{\phi \tau \beta-\tau \frac{\mu \gamma}{\kappa}+\tau\left(\alpha+\mu^{2} \gamma\right)} .
$$

Since the conjecture is $p=\kappa(\lambda y+\mu \theta-s)$, rational expectations imply that $\kappa^{-1}=\phi \tau \beta-\tau \mu \gamma / \kappa+$ $\tau\left(\alpha+\mu^{2} \gamma\right), \lambda=\tau(\alpha-\mu \gamma \lambda)$, and $\mu=\phi \tau \beta$. Thus, we obtain $\kappa=\left(1+\tau^{2} \phi \beta \gamma\right)\left[\phi \tau \beta+\tau \alpha+\tau(\phi \tau \beta)^{2} \gamma\right]^{-1}$, $\lambda=\tau \alpha /\left(1+\tau^{2} \phi \beta \gamma\right)$, and $\mu=\phi \tau \beta$. Therefore, it is easy to verify that $\kappa \lambda=\alpha /\left[\phi \beta+\alpha+(\phi \tau \beta)^{2} \gamma\right] \equiv$ $K(\phi)$ and $\kappa \mu=1-K(\phi)$ so $p=K(\phi) y+(1-K(\phi)) \theta-\kappa s$, where $K(\phi)$ is decreasing. It is now easy to verify that the asset demands by informed and uninformed traders are

$$
X_{i}=\tau \beta\left(x_{i}-p\right)+\frac{\tau \alpha}{1+\phi \tau^{2} \beta \gamma}(y-p), X=\frac{\tau \alpha}{1+\phi \tau^{2} \beta \gamma}(y-p)
$$

respectively. 


\section{References}

[1] Abreu, Dilip, and Markus K. Brunnermeier. "Bubbles and Crashes," Econometrica 71 (2003) $173-204$.

[2] Allen, Franklin, and Gary Gorton. "Churning Bubbles," Review of Economic Studies 60 (1993) $813-836$.

[3] Allen, Franklin, Stephen Morris, and Andrew Postlewaite. "Finite Bubbles with Short Sale Constraints and Asymmetric Information," Journal of Economic Theory 61 (1993) 206-229.

[4] Allen, Franklin, Stephen Morris, and Hyun Song Shin. "Beauty Contests and Iterated Expectations in Asset Markets," Review of Financial Studies 19 (2006) 719-752.

[5] Avery, Christopher, and Peter Zemsky. "Multidimensional Uncertainty and Herd Behavior in Financial Markets," American Economic Review 88 (1998) 724-748.

[6] Bacchetta, Philippe, and Eric Van Wincoop. "Higher Order Expectations in Asset Pricing," Journal of Money, Credit, and Banking 40 (2008) 837-866.

[7] Barlevy, Gadi, and Pietro Veronesi. "Rational Panics and Stock Market Crashes," Journal of Economic Theory 110 (2003) 234-263.

[8] Brown, David P., and Robert H. Jennings. "On Technical Analysis," Review of Financial Studies 2 (1989) 527-551.

[9] Brunnermeier, Markus K. Asset Pricing under Asymmetric Information: Bubbles, Crashes, Technical Analysis, and Herding, Oxford University Press, Oxford, 2001.

[10] Cespa, Giovanni. "Short-term Investment and Equilibrium Multiplicity," European Economic Review 46 (2002) 1645-1670.

[11] Cespa, Giovanni, and Xavier Vives. "Dynamic Trading and Asset Prices: Keynes vs. Hayek," mimeo, 2007. 
[12] Chamley, Christophe P. Rational Herds: Economic Models of Social Learning, Cambridge University Press, Cambridge, 2004.

[13] Chamley, Christophe P., and Douglas Gale. "Information Revelation and Strategic Delay in a Model of Investment," Econometrica 62 (1994) 1065-1085.

[14] Chari, V.V., and Patrick J. Kehoe. "Financial Crises as Herds: Overturning the Critiques," Journal of Economic Theory 119 (2004) 128-150.

[15] De Long, J. Bradford, Shleifer, Andrei, Lawrence H. Summers, and Robert J. Waldman. "Noise Trader Risk in Financial Markets," Journal of Political Economy 98 (1990) 703-738.

[16] Diamond, Douglas W., and Robert E. Verrecchia. "Information Aggregation in a Noisy Rational Expectations Economy," Journal of Financial Economics 9 (1981) 221-235.

[17] Gennotte, Gerard, and Hayne Leland. "Market Liquidity, Hedging, and Crashes," American Economic Review 80 (1990) 999-1021.

[18] Grossman, Sanford J. "On the Efficiency of Competitive Stock Markets where Trades Have Diverse Information," Journal of Finance 31 (1976) 573-585.

[19] Grossman, Sanford J., and Joseph E. Stiglitz. "On the Impossibility of Informationally Efficient Markets," American Economic Review 70 (1980) 393-408.

[20] Grundy, Bruce D., and Maureen McNichols. "Trade and the Revelation of Information through Prices and Direct Disclosure," Review of Financial Studies 2 (1990) 495-526.

[21] He, Hua, and Jiang Wang. "Differential Information and Dynamic Behavior of Stock Trading Volume," Review of Financial Studies 8 (1995) 919-972.

[22] Hellwig, Martin F. "On the Aggregation of Information in Competitive Markets," Journal of Economic Theory 22 (1980) 477-498.

[23] Keynes, John Maynard. The General Theory of Employment, Interest, and Money, Macmillan, London, 1936. 
[24] Kyle, Albert S. "Continuous Auctions and Insider Trading," Econometrica 53 (1985) 13151335.

[25] Kyle, Albert S. "Informed Speculation with Imperfect Competition," Review of Economic Studies 56 (1989) 317-536.

[26] LeRoy, Stephen F. "Efficient Capital Markets and Martingales," Journal of Economic Literature 27 (1989) 1583-1621.

[27] O’Hara, Maureen. Market Microstructure Theory, Blackwell, Oxford, 1995.

[28] Romer, David. "Rational Asset-Price Movements Without News," American Economic Review 83 (1993) 1112-1130.

[29] Samuelson, Paul A. "An Exact Consumption-Loan Model of Interest with or without the Social Contrivance of Money," Journal of Political Economy 66 (1958) 467-482.

[30] Tirole, Jean. "On the Possibility of Speculation under Rational Expectations," Econometrica 50 (1982) 1163-1181.

[31] Tirole, Jean. "Asset Bubbles and Overlapping Generations," Econometrica 53 (1985) 14991528.

[32] Vives, Xavier. "Short-Term Investment and the Informational Efficiency of the Market," Review of Financial Studies 8 (1995) 125-160.

[33] Vives, Xavier. Information and Learning in Markets: The Impact of Market Microstructure, Princeton University Press, New Jersey, 2008.

[34] Wang, Jiang. "A Model of Intertemporal Asset Prices Under Asymmetric Information," Review of Economic Studies 60 (1993) 249-282. 
Figure 1

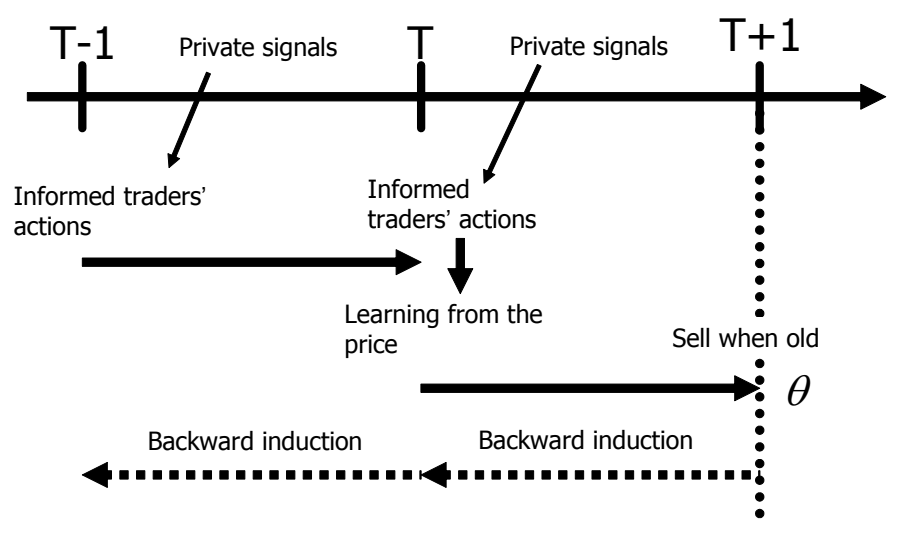


Figure 2: $y=1, \tau=1, \alpha=1, \beta=1, \gamma=1$

(a)

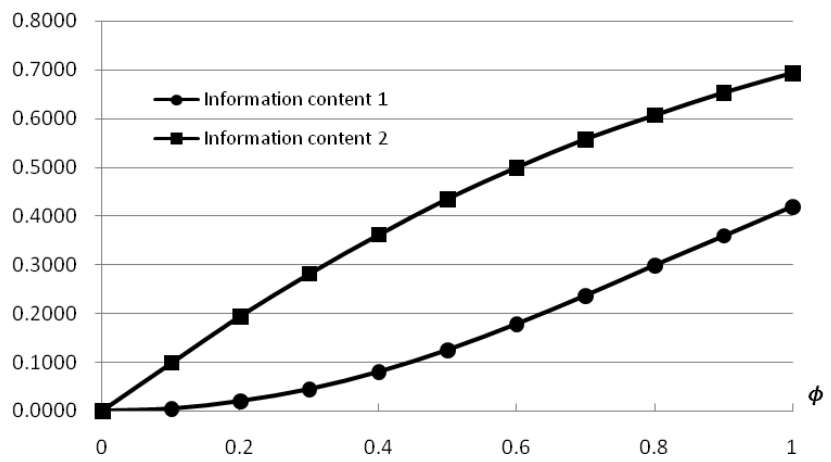

(b)

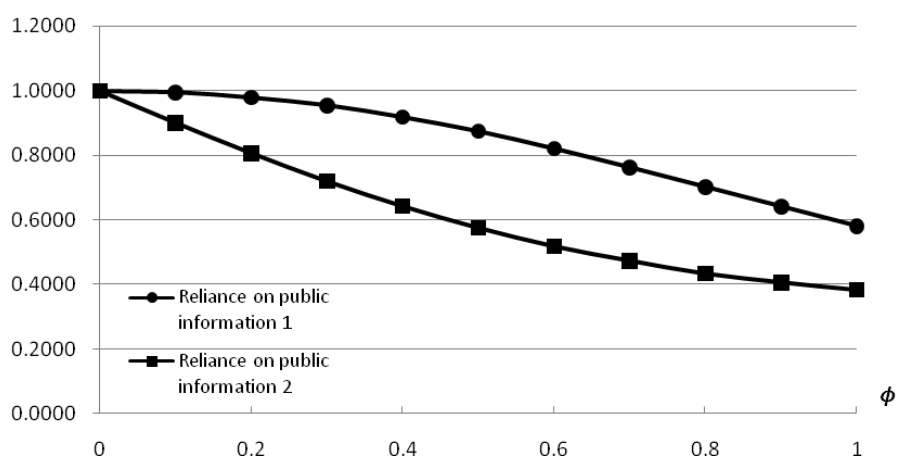

(c)

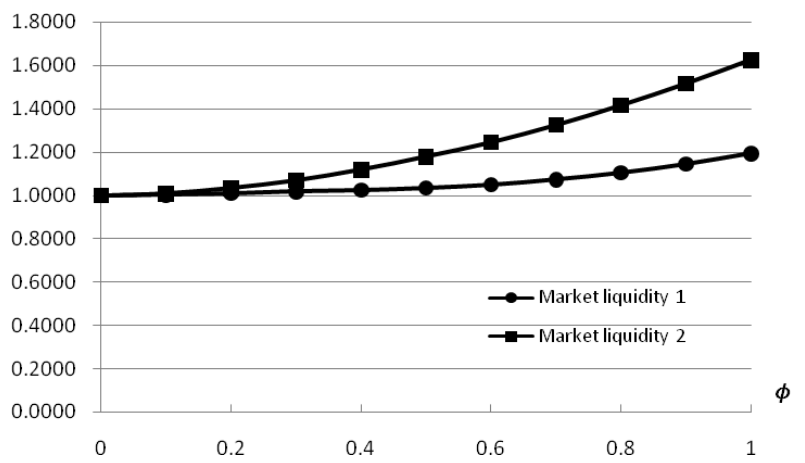

(d)

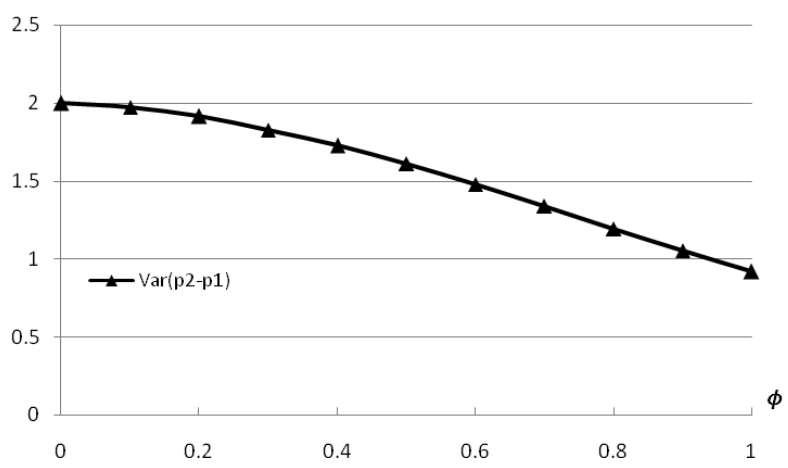


Figure 3: $y=1, \tau=1, \alpha=2, \beta=3, \gamma=1$

(a)

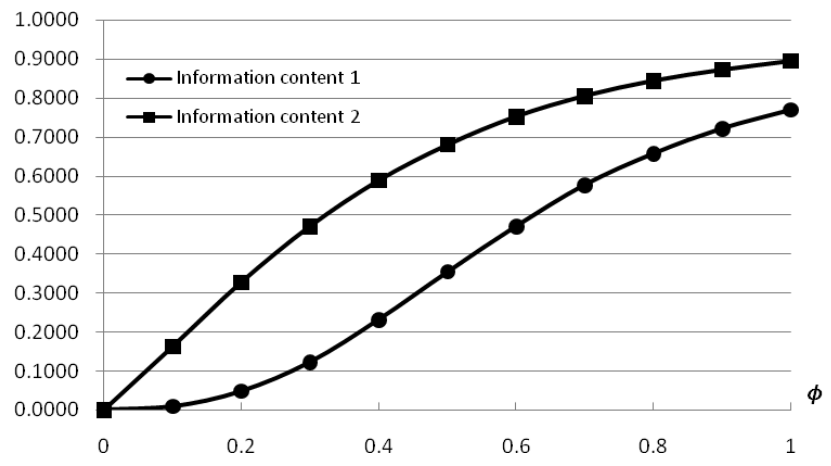

(b)

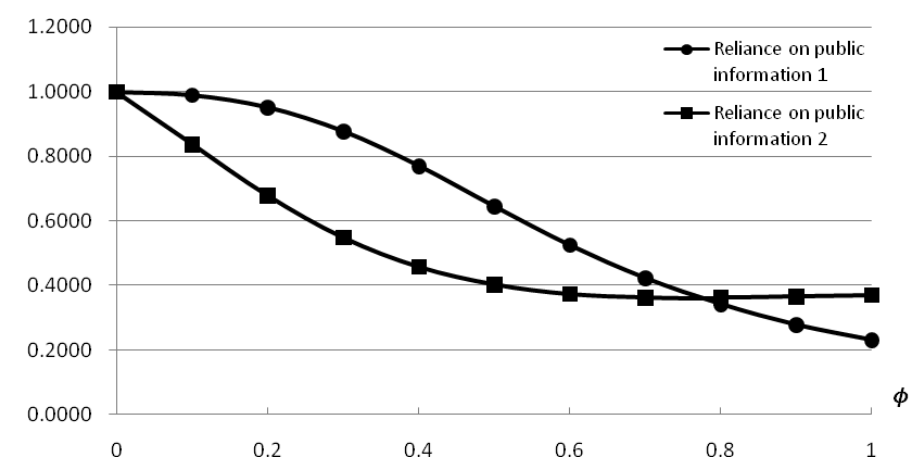

(c)

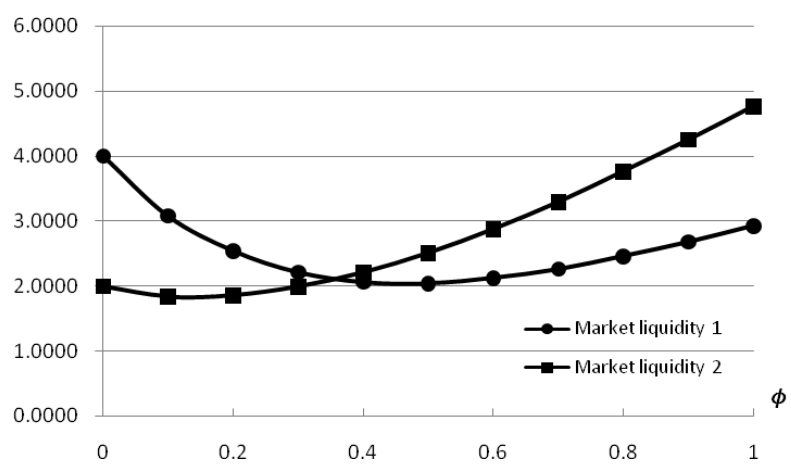

(d)

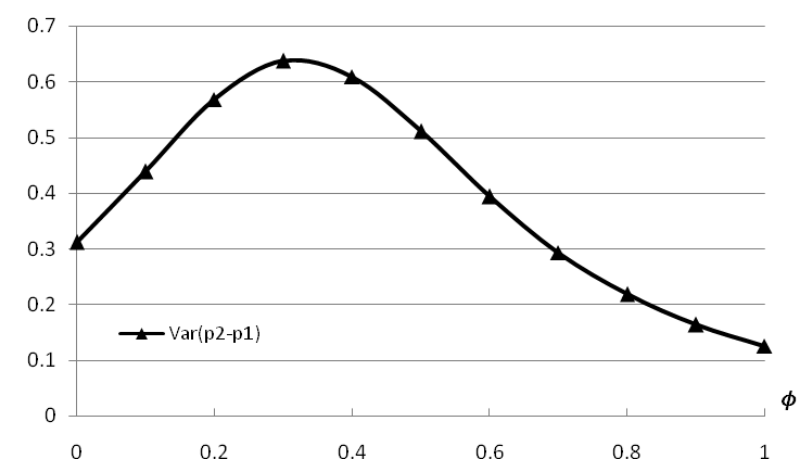


Figure 4: $y=1, \tau=1, \alpha=2, \beta=0.5, \gamma=1$

(a)

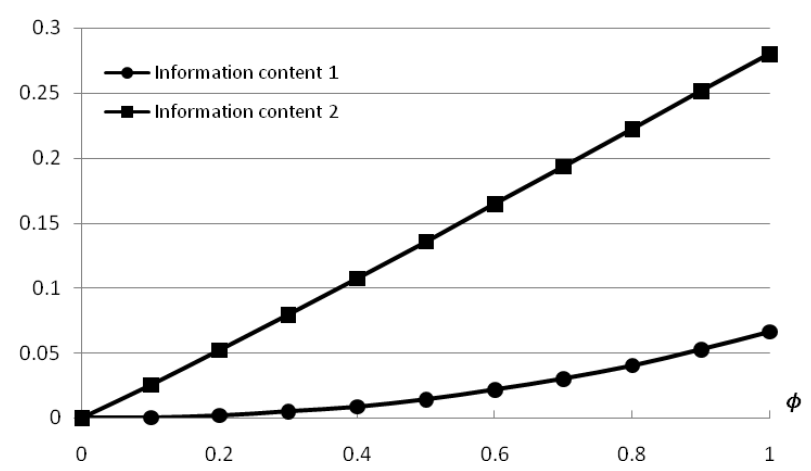

(b)

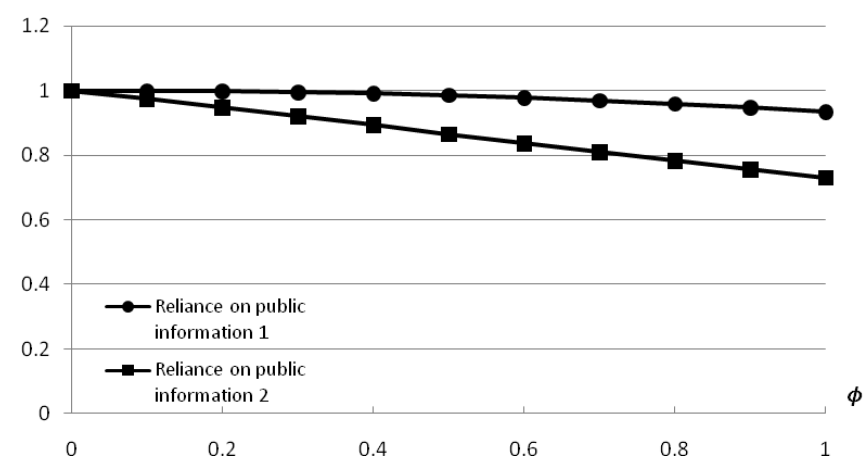

(c)

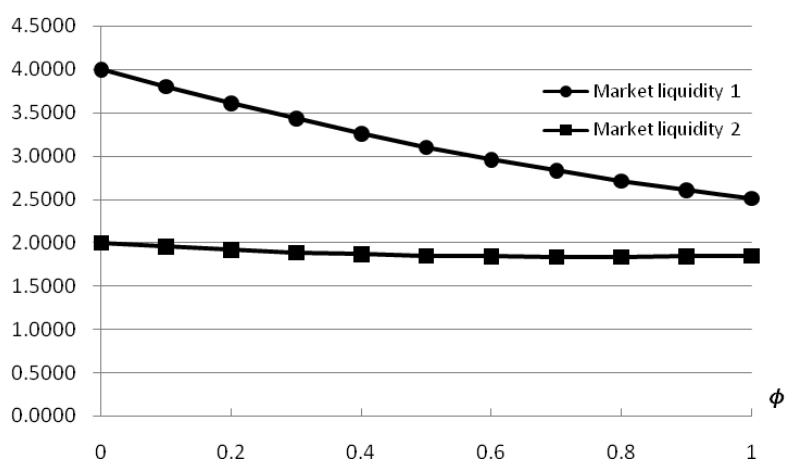

(d)

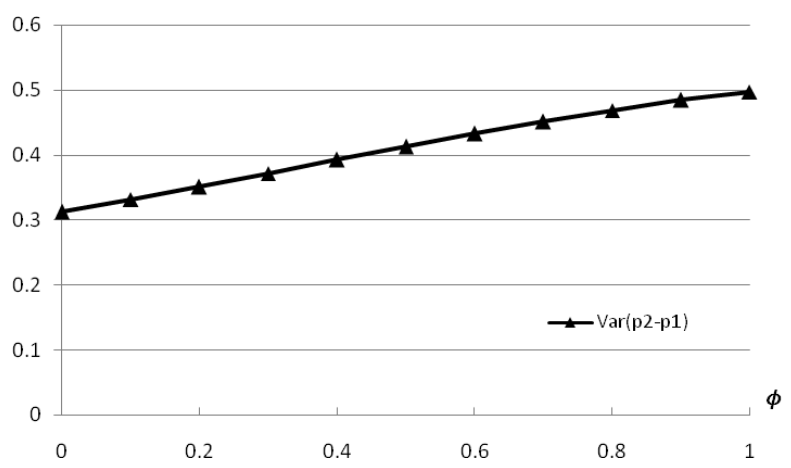


Figure 5: Market Orders $(y=1, \tau=1, \alpha=1, \beta=1, \gamma=1)$

(a)

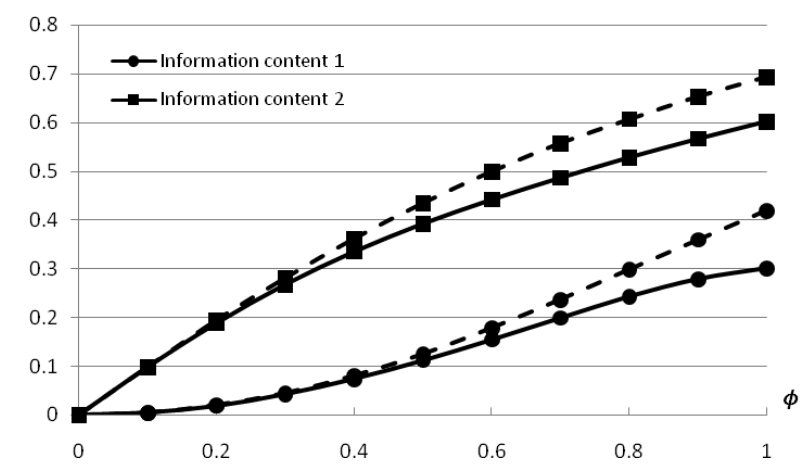

(b)

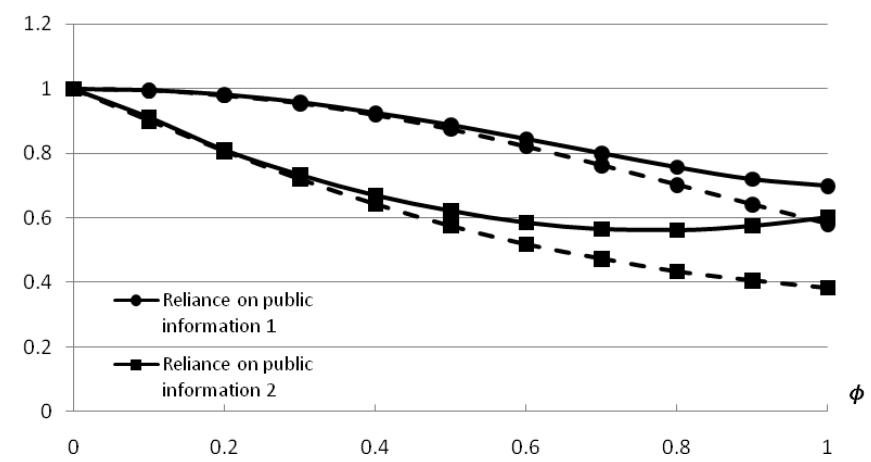

(c)

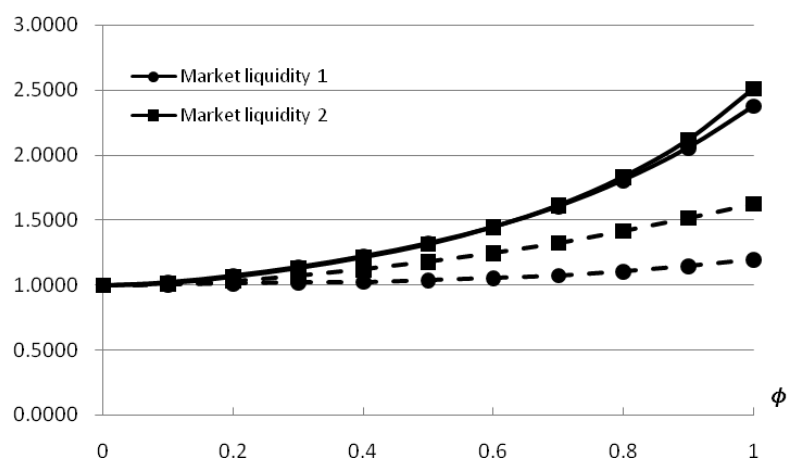

(d)

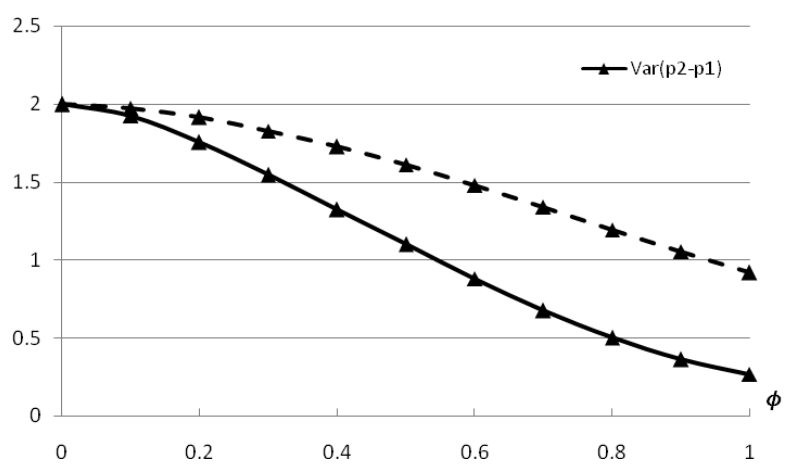


Figure 6: Market Orders $(y=1, \tau=1, \alpha=2, \beta=3, \gamma=1)$

(a)

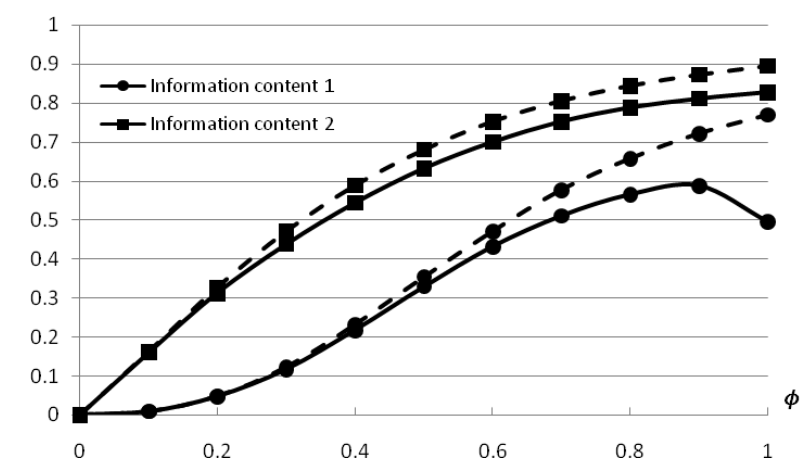

(b)

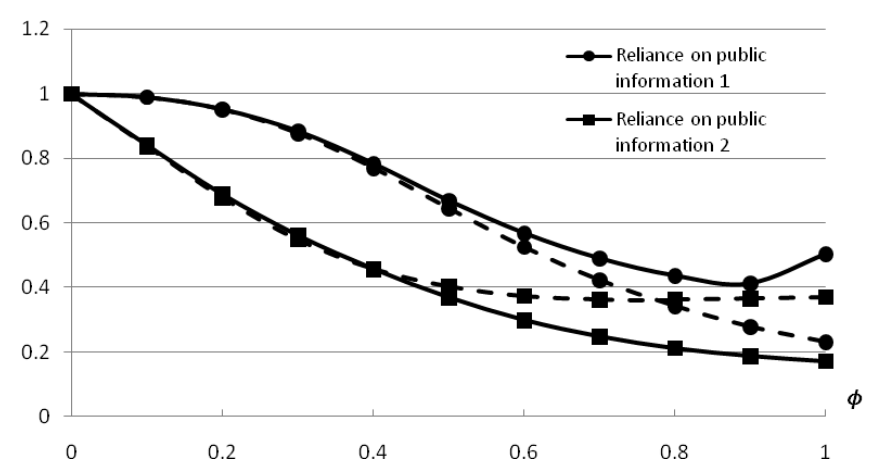

(c)

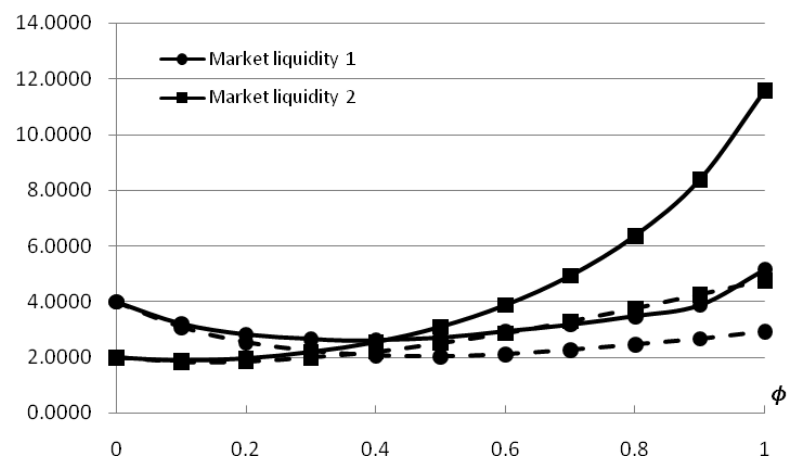

(d)

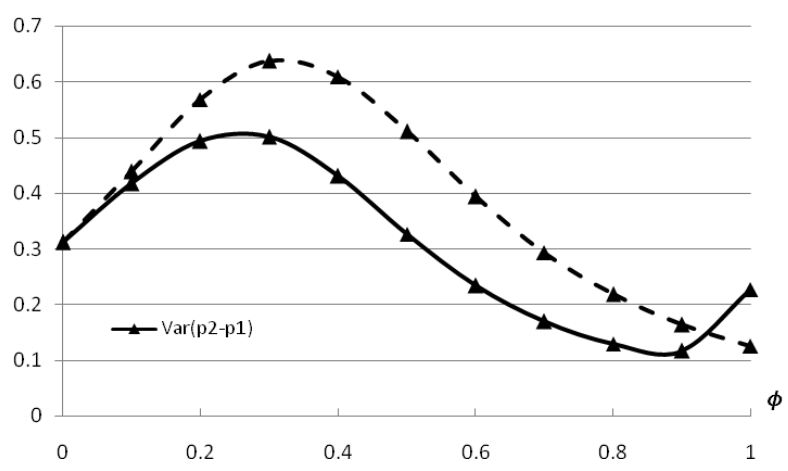

